\title{
Renormalized Electron Mass in Nonrelativistic QED
}

\author{
Jürg Fröhlich ${ }^{1, \star}$, Alessandro Pizzo ${ }^{2}$ \\ 1 Institute of Theoretical Physics, ETH Zürich, CH-8093 Zürich, \\ Switzerland. E-mail: juerg@itp.phys.ethz.ch \\ 2 Department of Mathematics, University of California Davis, \\ One Shields Avenue, Davis, California 95616, USA. \\ E-mail:pizzo@math.ucdavis.edu
}

Received: 5 February 2009 / Accepted: 27 July 2009

Published online: 5 December 2009 - (C) The Author(s) 2009. This article is published with open access at Springerlink.com

\begin{abstract}
Within the framework of nonrelativistic QED, we prove that, for small values of the coupling constant, the energy function, $E_{\vec{P}}$, of a dressed electron is twice differentiable in the momentum $\vec{P}$ in a neighborhood of $\vec{P}=0$. Furthermore, $\frac{\partial^{2} E_{\vec{P}}}{(\partial|\vec{P}|)^{2}}$ is bounded from below by a constant larger than zero. Our results are proven with the help of iterative analytic perturbation theory.
\end{abstract}

\section{Description of the Problem, Definition of the Model, and Outline of the Proof}

In this paper, we study problems connected with the renormalized electron mass in a model of quantum electrodynamics (QED) with nonrelativistic matter. We are interested in rigorously controlling radiative corrections to the electron mass caused by the interaction of the electron with the soft modes of the quantized electromagnetic field. The model describing interactions between nonrelativistic, quantum-mechanical charged matter and the quantized radiation field at low energies (i.e., energies smaller than the rest energy of an electron) is the "standard model", see [7]. In this paper, we consider a system consisting of a single spinless electron, described as a nonrelativistic particle that is minimally coupled to the quantized radiation field, and photons. Electron spin can easily be included in our description without substantial complications.

The physical system studied in this paper exhibits space translations invariance. The Hamiltonian, $H$, generating the time evolution, commutes with the vector operator, $\vec{P}$, representing the total momentum of the system, which generates space translations. If an infrared regularization, e.g., an infrared cutoff $\sigma$ on the photon frequency, is imposed on the interaction Hamiltonian, there exist single-electron or dressed one-electron states, as long as their momentum is smaller than the bare electron mass, $m$, of the electron. This means that a notion of mass shell in the energy momentum spectrum is meaningful for

^ Also at IHES, Bures-sur-Yvette. 
velocities $|\vec{P}| / m$ smaller than the speed of light $c$; (with $c \equiv m \equiv 1$ in our units). Vectors $\left\{\Psi^{\sigma}\right\}$ describing dressed one-electron states are normalizable vectors in the Hilbert space $\mathcal{H}$ of pure states of the system. They are characterized as solutions of the equation

$$
H^{\sigma} \Psi^{\sigma}=E_{\vec{P}}^{\sigma} \Psi^{\sigma},|\vec{P}|<1,
$$

where $H^{\sigma}$ is the Hamiltonian with an infrared cutoff $\sigma$ in the interaction term and $E_{\vec{P}}^{\sigma}$, the energy of a dressed electron, is a function of the momentum operator $\vec{P}$. If in the joint spectrum of the components of $\vec{P}$ the support of the vector $\Psi^{\sigma}$ is contained in a ball centered at the origin and of radius less than $1 \equiv m c$, then Eq. (I.1) has solutions; see [6]. Since $[H, \vec{P}]=0$, Eq. (I.1) can be studied for the fiber vectors, $\Psi_{\vec{P}}^{\sigma}$, corresponding to a value, $\vec{P}$, of the total momentum (both the total momentum operator and points in its spectrum will henceforth be denoted by $\vec{P}$ - without danger of confusion). Thus we consider the equation

$$
H_{\vec{P}}^{\sigma} \Psi_{\vec{P}}^{\sigma}=E_{\vec{P}}^{\sigma} \Psi_{\vec{P}}^{\sigma}
$$

where $H_{\vec{P}}^{\sigma}$ is the fiber Hamiltonian at fixed total momentum $\vec{P}$, and $E_{\vec{P}}^{\sigma}$ is the value of the function $E_{\vec{z}}^{\sigma}$ at the point $\vec{z} \equiv \vec{P}$. Physically, states $\left\{\Psi^{\sigma}\right\}$ solving Eq. (I.1) describe a freely moving electron in the absence of asymptotic photons.

It is an essential aspect of the "infrared catastrophe" in QED that Eq. (I.1) does not have any normalizable solution in the limit where the infrared cut-off $\sigma$ tends to zero, and the underlying dynamical picture of a freely moving electron breaks down; see [5]. Nevertheless, the limiting behavior of the function $E_{\vec{p}}^{\sigma}$ is of great interest for the following reasons.

As long as $\sigma>0$, a natural definition of the renormalized electron mass, $m_{r}$, is given by the formula

$$
m_{r}(\sigma):=\left[\left.\frac{\partial^{2} E_{|\vec{P}|}^{\sigma}}{(\partial|\vec{P}|)^{2}}\right|_{\vec{P}=0}\right]^{-1} .
$$

(Note that $E_{\vec{P}}^{\sigma} \equiv E_{|\vec{P}|}^{\sigma}$ is invariant under rotations.) Equation (I.3) is expected to remain meaningful in the limit $\sigma \rightarrow 0$. In particular, the quantity on the R.H.S. of Eq. (I.3) is expected to be positive and bounded from above uniformly in the infrared cutoff $\sigma$.

More importantly, one aims at mathematical control of the function

$$
m_{r}(\sigma,|\vec{P}|):=\left[\frac{\partial^{2} E_{|\vec{P}|}^{\sigma}}{(\partial|\vec{P}|)^{2}}\right]^{-1}
$$

in a full neighborhood, $\mathcal{S}$, of $\vec{P}=0$, corresponding to a slowly moving electron (i.e., in the nonrelativistic regime). When combined with a number of other spectral properties of the Hamiltonian of nonrelativistic QED the condition

$$
\frac{\partial^{2} E_{|\vec{P}|}^{\sigma}}{(\partial|\vec{P}|)^{2}}>0 \quad, \quad \vec{P} \in \mathcal{S},
$$


uniformly in $\sigma>0$, suffices to yield a consistent scattering picture in the limit when $\sigma \rightarrow 0$ in which the electron exhibits infraparticle behavior. In fact, (I.5) is a crucial ingredient in the analysis of Compton scattering presented in $[5,13]$.

Main results. Assuming the coupling constant, $\alpha$, small enough, the following results follow.

1) The function

$$
\Sigma_{|\vec{P}|}:=\lim _{\sigma \rightarrow 0} \frac{\partial^{2} E_{|\vec{P}|}^{\sigma}}{(\partial|\vec{P}|)^{2}}
$$

is well defined for $\vec{P} \in \mathcal{S}:=\left\{\vec{P}|| \vec{P} \mid<\frac{1}{3}\right\}$; furthermore, it is Hölder-continuous in $\vec{P}$.

2) The function

$$
E_{\vec{P}}:=\lim _{\sigma \rightarrow 0} E_{\vec{P}}^{\sigma}
$$

is twice differentiable in $\vec{P} \in \mathcal{S}$ and

$$
\frac{\partial^{2} E_{|\vec{P}|}}{(\partial|\vec{P}|)^{2}}=\Sigma_{|\vec{P}|} .
$$

3)

$$
\lim _{\alpha \rightarrow 0} \frac{\partial^{2} E_{|\vec{P}|}^{\sigma}}{(\partial|\vec{P}|)^{2}}=\frac{1}{m} \quad, \quad \vec{P} \in \mathcal{S},
$$

uniformly in $\sigma$, where $m$ is the bare electron mass. (Our results can be extended to a region $\mathcal{S}$ (inside the unit ball) of radius larger than $\frac{1}{3}$.)

We wish to mention some related earlier results. Using operator-theoretic renormalization group methods, results (I.6) and (I.9) have been proven in [2] for the special value $\vec{P}=0$. The point $\vec{P}=0$ is exceptional, because the Hamiltonian $H_{\vec{P}}$ is infrared regular at $\vec{P}=0$; it has a normalizable ground state. Thomas Chen (see [3]) has established the results in (I.8), (I.9) (using smooth infrared cut-offs) by a highly non-trivial extension of the analysis of [2] to arbitrary momenta $\vec{P} \in \mathcal{S}$.

The procedure presented in our paper relies on iterative analytic perturbation theory (see Sect. II where this tool is recalled) that makes our proof substantially different and much shorter in comparison to a renormalization group approach. The main feature is a more transparent treatment of the so-called marginal terms of the interaction, where an essential role is played by explicit Bogoliubov transformations that transform the infrared representations of the CCR of photon creation- and annihilation operators determined by dressed one-particle states of fixed momentum $\vec{P}(\neq 0)$ back to the Fock representation. The use of these Bogoliubov transformations is a crucial device in our fight against the infrared problem. The way in which we are using them is new, at least in the context of mathematically rigorous results on the infrared problem in QED.

In our paper, the regularity properties of $E_{\vec{P}}$ come with an explicit control of the asymptotics of the fiber ground state eigenvectors $\Psi_{\vec{P}}^{\sigma}$ as $\sigma$ tends to zero. (This improves earlier results in [6].) Along the lines of [1], these results are preparatory to developing an infrared finite algorithm for the asymptotic expansion of the renormalized electron mass 
in powers and, probably, logarithms of the finestructure constant $\alpha$, up to an arbitrarily small remainder term. However, the expansion in the coupling constant $\alpha$ is not studied in this paper.

With regard to ultraviolet corrections to the electron mass in nonrelativistic QED models, we refer the reader to [8,10,11 and 9].

In Sect. I.1 below, the model is defined rigorously. Then, for the convenience of the reader, in Sect. I. 2 we outline the key ideas of the proof and present the organization of the remaining sections of the paper.

\section{I.1. Definition of the model.}

Hilbert space. The Hilbert space of pure state vectors of a system consisting of one non-relativistic electron interacting with the quantized electromagnetic field is given by

$$
\mathcal{H}:=\mathcal{H}_{e l} \otimes \mathcal{F}
$$

where $\mathcal{H}_{e l}=L^{2}\left(\mathbb{R}^{3}\right)$ is the Hilbert space for a single Schrödinger electron; for expository convenience, we neglect the spin of the electron. The Hilbert space, $\mathcal{F}$, used to describe the states of the transverse modes of the quantized electromagnetic field (the photons) in the Coulomb gauge is given by the Fock space

$$
\mathcal{F}:=\bigoplus_{N=0}^{\infty} \mathcal{F}^{(N)}, \quad \mathcal{F}^{(0)}=\mathbb{C} \Omega,
$$

where $\Omega$ is the vacuum vector (the state of the electromagnetic field without any excited modes), and

$$
\mathcal{F}^{(N)}:=\mathcal{S}_{N} \bigotimes_{j=1}^{N} \mathfrak{h}, \quad N \geq 1,
$$

where the Hilbert space $\mathfrak{h}$ of state vectors of a single photon is

$$
\mathfrak{h}:=L^{2}\left(\mathbb{R}^{3} \times \mathbb{Z}_{2}\right) \text {. }
$$

Here, $\mathbb{R}^{3}$ is momentum space, and $\mathbb{Z}_{2}$ accounts for the two independent transverse polarizations (or helicities) of a photon. In (I.12), $\mathcal{S}_{N}$ denotes the orthogonal projection onto the subspace of $\bigotimes_{j=1}^{N} \mathfrak{h}$ of totally symmetric $N$-photon wave functions, which accounts for the fact that photons satisfy Bose-Einstein statistics. Thus, $\mathcal{F}^{(N)}$ is the subspace of $\mathcal{F}$ of state vectors corresponding to configurations of exactly $N$ photons.

Units. In this paper, we employ units such that Planck's constant $\hbar$, the speed of light $c$, and the mass of the electron $m$ are equal to 1 .

Hamiltonian. The dynamics of the system is generated by the Hamiltonian

$$
H:=\frac{\left(-i \vec{\nabla}_{\vec{x}}+\alpha^{1 / 2} \vec{A}(\vec{x})\right)^{2}}{2}+H^{f} .
$$

The (three-component) multiplication operator $\vec{x} \in \mathbb{R}^{3}$ represents the position of the electron. The electron momentum operator is given by $\vec{p}=-i \vec{\nabla} \vec{x}$. Furthermore, $\alpha>0$ 
is the fine structure constant (which, in this paper, plays the rôle of a small parameter), and $\vec{A}(\vec{x})$ denotes the vector potential of the transverse modes of the quantized electromagnetic field in the Coulomb gauge,

$$
\vec{\nabla}_{\vec{x}} \cdot \vec{A}(\vec{x})=0
$$

cutoff at high photon frequencies.

$H^{f}$ is the Hamiltonian of the quantized, free electromagnetic field. It is given by

$$
H^{f}:=\sum_{\lambda= \pm} \int d^{3} k|\vec{k}| a_{\vec{k}, \lambda}^{*} a_{\vec{k}, \lambda}
$$

where $a_{\vec{k}, \lambda}^{*}$ and $a_{\vec{k}, \lambda}$ are the usual photon creation- and annihilation operators satisfying the canonical commutation relations

$$
\begin{aligned}
& {\left[a_{\vec{k}, \lambda}, a_{\vec{k}^{\prime}, \lambda^{\prime}}^{*}\right]=\delta_{\lambda \lambda^{\prime}} \delta\left(\vec{k}-\vec{k}^{\prime}\right),} \\
& {\left[a_{\vec{k}, \lambda}^{\#}, a_{\vec{k}^{\prime}, \lambda^{\prime}}^{\#}\right]=0,}
\end{aligned}
$$

for $\vec{k}, \vec{k}^{\prime} \in \mathbb{R}^{3}$ and $\lambda, \lambda^{\prime} \in \mathbb{Z}_{2} \equiv\{ \pm\}$, where $a^{\#}=a$ or $a^{*}$. The vacuum vector $\Omega \in \mathcal{F}$ is characterized by the condition

$$
a_{\vec{k}, \lambda} \Omega=0
$$

for all $\vec{k} \in \mathbb{R}^{3}$ and $\lambda \in \mathbb{Z}_{2} \equiv\{ \pm\}$.

The quantized electromagnetic vector potential is given by

$$
\vec{A}(\vec{x}):=\sum_{\lambda= \pm} \int_{\mathcal{B}_{\Lambda}} \frac{d^{3} k}{\sqrt{|\vec{k}|}}\left\{\vec{\varepsilon}_{\vec{k}, \lambda} e^{-i \vec{k} \cdot \vec{x}} a_{\vec{k}, \lambda}^{*}+\vec{\varepsilon}_{\vec{k}, \lambda}^{*} e^{i \vec{k} \cdot \vec{x}} a_{\vec{k}, \lambda}\right\},
$$

where $\vec{\varepsilon}_{\vec{k},-}, \vec{\varepsilon}_{\vec{k},+}$ are photon polarization vectors, i.e., two unit vectors in $\mathbb{R}^{3} \otimes \mathbb{C}$ satisfying

$$
\vec{\varepsilon}_{\vec{k}, \lambda}^{*} \cdot \vec{\varepsilon}_{\vec{k}, \mu}=\delta_{\lambda \mu}, \quad \vec{k} \cdot \vec{\varepsilon}_{\vec{k}, \lambda}=0
$$

for $\lambda, \mu= \pm$. The equation $\vec{k} \cdot \vec{\varepsilon}_{\vec{k}, \lambda}=0$ expresses the Coulomb gauge condition. Moreover, $\mathcal{B}_{\Lambda}$ is a ball of radius $\Lambda$ centered at the origin in momentum space. Here, $\Lambda$ represents an ultraviolet cutoff that will be kept fixed throughout our analysis. The vector potential defined in (I.20) is thus cut off in the ultraviolet.

Throughout this paper, it will be assumed that $\Lambda \approx 1$ (the rest energy of an electron), and that $\alpha>0$ is sufficiently small. Under these assumptions, the Hamiltonian $H$ is selfadjoint on $D\left(H^{0}\right)$, i.e., on the domain of definition of the operator

$$
H^{0}:=\frac{(-i \vec{\nabla} \vec{x})^{2}}{2}+H^{f}
$$

The perturbation $H-H^{0}$ is small in the sense of Kato.

The operator representing the total momentum of the system consisting of the electron and the electromagnetic radiation field is given by

$$
\vec{P}:=\vec{p}+\vec{P}^{f}
$$


with $\vec{p}=-i \vec{\nabla}_{\vec{x}}$, and where

$$
\vec{P}^{f}:=\sum_{\lambda= \pm} \int d^{3} k \vec{k} a_{\vec{k}, \lambda}^{*} a_{\vec{k}, \lambda}
$$

is the momentum operator associated with the photon field.

The operators $H$ and $\vec{P}$ are essentially selfadjoint on a common domain, and since the dynamics is invariant under translations, they commute, $[H, \vec{P}]=\overrightarrow{0}$. The Hilbert space $\mathcal{H}$ can be decomposed into a direct integral over the joint spectrum, $\mathbb{R}^{3}$, of the three components of the momentum operator $\vec{P}$. Their spectral measure is absolutely continuous with respect to Lebesgue measure, and hence we have that

$$
\mathcal{H}:=\int^{\oplus} \mathcal{H}_{\vec{P}} d^{3} P
$$

where each fiber space $\mathcal{H}_{\vec{P}}$ is a copy of Fock space $\mathcal{F}$.

Remark. Throughout this paper, the symbol $\vec{P}$ stands for both a vector in $\mathbb{R}^{3}$ and the vector operator on $\mathcal{H}$, representing the total momentum, depending on context. Similarly, a double meaning is given to arbitrary functions, $f(\vec{P})$, of the total momentum operator.

We recall that vectors $\Psi \in \mathcal{H}$ are given by sequences

$$
\left\{\Psi^{(m)}\left(\vec{x} ; \vec{k}_{1}, \lambda_{1} ; \ldots ; \vec{k}_{m}, \lambda_{m}\right)\right\}_{m=0}^{\infty},
$$

of functions, $\Psi^{(m)}$, where $\Psi^{(0)}(\vec{x}) \in L^{2}\left(\mathbb{R}^{3}\right)$, of the electron position $\vec{x}$ and of $m$ photon momenta $\vec{k}_{1}, \ldots, \vec{k}_{m}$ and helicities $\lambda_{1}, \ldots, \lambda_{m}$, with the following properties:

(i) $\Psi^{(m)}\left(\vec{x} ; \vec{k}_{1}, \lambda_{1} ; \ldots ; \vec{k}_{m}, \lambda_{m}\right)$ is totally symmetric in its $m$ arguments $\left(\vec{k}_{j}, \lambda_{j}\right)_{j=1, \ldots, m}$

(ii) $\Psi^{(m)}$ is square-integrable, for all $m$,

(iii) If $\Psi$ and $\Phi$ are two vectors in $\mathcal{H}$, then

$$
\begin{aligned}
& (\Psi, \Phi)=\sum_{m=0}^{\infty}\left(\sum_{\lambda_{j}= \pm} \int d^{3} x \prod_{j=1}^{m} d^{3} k_{j} \overline{\Psi^{(m)}\left(\vec{x} ; \vec{k}_{1}, \lambda_{1} ; \ldots ; \vec{k}_{m}, \lambda_{m}\right)}\right. \\
& \left.\times \Phi^{(m)}\left(\vec{x} ; \vec{k}_{1}, \lambda_{1} ; \ldots ; \vec{k}_{m}, \lambda_{m}\right)\right) \text {. }
\end{aligned}
$$

We identify a square integrable function $g(\vec{x})$ with the sequence

$$
\left\{\Psi^{(m)}\left(\vec{x} ; \vec{k}_{1}, \lambda_{1} ; \ldots ; \vec{k}_{m}, \lambda_{m}\right)\right\}_{m=0}^{\infty},
$$

where $\Psi^{(0)}(\vec{x}) \equiv g(\vec{x})$, and $\Psi^{(m)}\left(\vec{x} ; \vec{k}_{1}, \lambda_{1} ; \ldots ; \vec{k}_{m}, \lambda_{m}\right) \equiv 0$ for all $m>0$; analogously, a square integrable function $g^{(m)}\left(\vec{x} ; \vec{k}_{1}, \lambda_{1} ; \ldots ; \vec{k}_{m}, \lambda_{m}\right), m \geq 1$, is identified with the sequence

$$
\left\{\Psi^{\left(m^{\prime}\right)}\left(\vec{x} ; \vec{k}_{1}, \lambda_{1} ; \ldots ; \vec{k}_{m^{\prime}}, \lambda_{m^{\prime}}\right)\right\}_{m^{\prime}=0}^{\infty},
$$


where $\Psi^{(m)}\left(\vec{x} ; \vec{k}_{1}, \lambda_{1} ; \ldots ; \vec{k}_{m}, \lambda_{m}\right) \equiv g^{(m)}$, and $\Psi^{\left(m^{\prime}\right)}\left(\vec{x} ; \vec{k}_{1}, \lambda_{1} ; \ldots ; \vec{k}_{m^{\prime}}, \lambda_{m^{\prime}}\right) \equiv 0$ for all $m^{\prime} \neq m$. From now on, a sequence describing a quantum state with a fixed number of photons is identified with its nonzero component wave function; vice versa, a wave function corresponds to a sequence according to the previous identification. The elements of the fiber space $\mathcal{H}_{\vec{P}^{*}}$ are obtained by linear combinations of the (improper) eigenvectors of the total momentum operator $\vec{P}$ with eigenvalue $\vec{P}^{*}$, e.g., the plane wave $e^{i \vec{P}^{*} \cdot \vec{x}}$ is the eigenvector describing a state with an electron and no photon.

Given any $\vec{P} \in \mathbb{R}^{3}$, there is an isomorphism, $I_{\vec{P}}$,

$$
I_{\vec{P}}: \mathcal{H}_{\vec{P}} \longrightarrow \mathcal{F}^{b},
$$

from the fiber space $\mathcal{H}_{\vec{P}}$ to the Fock space $\mathcal{F}^{b}$, acted upon by the annihilation- and creation operators $b_{\vec{k}, \lambda}, b_{\vec{k}, \lambda}^{*}$, where $b_{\vec{k}, \lambda}$ corresponds to $e^{i \vec{k} \cdot \vec{x}} a_{\vec{k}, \lambda}$, and $b_{\vec{k}, \lambda}^{*}$ to $e^{-i \vec{k} \cdot \vec{x}} a_{\vec{k}, \lambda}^{*}$, and with vacuum $\Omega_{f}:=I_{\vec{P}}\left(e^{i \vec{P} \cdot \vec{x}}\right)$. To define $I_{\vec{P}}$ more precisely, we consider a vector $\psi_{\left(f^{(n)} ; \vec{P}\right)} \in \mathcal{H}_{\vec{P}}$ with a definite total momentum describing an electron and $n$ photons. Its wave function in the variables $\left(\vec{x} ; \vec{k}_{1}, \lambda_{1} ; \ldots, \vec{k}_{n}, \lambda_{n}\right)$ is given by

$$
e^{i\left(\vec{P}-\vec{k}_{1}-\cdots-\vec{k}_{n}\right) \cdot \vec{x}} f^{(n)}\left(\vec{k}_{1}, \lambda_{1} ; \ldots ; \vec{k}_{n}, \lambda_{n}\right),
$$

where $f^{(n)}$ is totally symmetric in its $n$ arguments. The isomorphism $I_{\vec{P}}$ acts by way of

$$
\begin{aligned}
& I_{\vec{P}}\left(e^{i\left(\vec{P}-\vec{k}_{1}-\ldots-\vec{k}_{n}\right) \cdot \vec{x}} f^{(n)}\left(\vec{k}_{1}, \lambda_{1} ; \ldots ; \vec{k}_{n}, \lambda_{n}\right)\right) \\
& \quad=\frac{1}{\sqrt{n !}} \sum_{\lambda_{1}, \ldots, \lambda_{n}} \int d^{3} k_{1} \ldots d^{3} k_{n} f^{(n)}\left(\vec{k}_{1}, \lambda_{1} ; \ldots ; \vec{k}_{n}, \lambda_{n}\right) b_{\vec{k}_{1}, \lambda_{1}}^{*} \cdots b_{\vec{k}_{n}, \lambda_{n}}^{*} \Omega_{f} .
\end{aligned}
$$

Because the Hamiltonian $H$ commutes with the total momentum, it preserves the fibers $\mathcal{H}_{\vec{P}}$ for all $\vec{P} \in \mathbb{R}^{3}$, i.e., it can be written as

$$
H=\int^{\oplus} H_{\vec{P}} d^{3} P
$$

where

$$
H_{\vec{P}}: \mathcal{H}_{\vec{P}} \longrightarrow \mathcal{H}_{\vec{P}}
$$

Written in terms of the operators $b_{\vec{k}, \lambda}, b_{\vec{k}, \lambda}^{*}$, and of the variable $\vec{P}$, the fiber Hamiltonian $H_{\vec{P}}$ is given by

$$
H_{\vec{P}}:=\frac{\left(\vec{P}-\vec{P}^{f}+\alpha^{1 / 2} \vec{A}\right)^{2}}{2}+H^{f},
$$

where

$$
\begin{aligned}
\vec{P}^{f} & =\sum_{\lambda} \int d^{3} k \vec{k} b_{\vec{k}, \lambda}^{*} b_{\vec{k}, \lambda}, \\
H^{f} & =\sum_{\lambda} \int d^{3} k|\vec{k}| b_{\vec{k}, \lambda}^{*} b_{\vec{k}, \lambda},
\end{aligned}
$$


and

$$
\vec{A}:=\sum_{\lambda} \int_{\mathcal{B}_{\Lambda}} \frac{d^{3} k}{\sqrt{|\vec{k}|}}\left\{\vec{\varepsilon}_{\vec{k}, \lambda} b_{\vec{k}, \lambda}^{*}+\vec{\varepsilon}_{\vec{k}, \lambda}^{*} b_{\vec{k}, \lambda}\right\} .
$$

Let

$$
\mathcal{S}:=\left\{\vec{P} \in \mathbb{R}^{3}:|\vec{P}|<\frac{1}{3}\right\}
$$

In order to give a mathematically precise meaning to the constructions presented in the following, we introduce an infrared cut-off at a photon frequency $\sigma>0$ in the vector potential. The calculation of the second derivative of the energy of a dressed electron in the following called the "ground state energy" - as a function of $\vec{P}$ in the limit where $\sigma \rightarrow 0$, and for $\vec{P} \in \mathcal{S}$, represents the main problem solved in this paper. Hence we will, in the sequel, study the regularized fiber Hamiltonian

$$
H_{\vec{P}}^{\sigma}:=\frac{\left(\vec{P}-\vec{P}^{f}+\alpha^{1 / 2} \vec{A}^{\sigma}\right)^{2}}{2}+H^{f},
$$

acting on the fiber space $\mathcal{H}_{\vec{P}}$, for $\vec{P} \in \mathcal{S}$, where

$$
\vec{A}^{\sigma}:=\sum_{\lambda} \int_{\mathcal{B}_{\Lambda} \backslash \mathcal{B}_{\sigma}} \frac{d^{3} k}{\sqrt{|\vec{k}|}}\left\{\vec{\varepsilon}_{\vec{k}, \lambda} b_{\vec{k}, \lambda}^{*}+\vec{\varepsilon}_{\vec{k}, \lambda}^{*} b_{\vec{k}, \lambda}\right\},
$$

and where $\mathcal{B}_{\sigma}$ is a ball of radius $\sigma$ centered at the origin. In the following, we will consider a sequence of infrared cutoffs

$$
\sigma_{j}:=\Lambda \epsilon^{j}
$$

with $0<\epsilon<\frac{1}{2}$ and $j \in \mathbb{N}_{0}:=\mathbb{N} \cup\{0\}$.

Notation. 1) We use the notation $\|A\|_{\mathcal{H}}=\left\|\left.A\right|_{\mathcal{H}}\right\|$ for the norm of a bounded operator $A$ acting on a Hilbert space $\mathcal{H}$. Typically, $\mathcal{H}$ will be some subspace of $\mathcal{F}^{b}$.

2) Throughout the paper, we follow conventions such that

$$
\frac{1}{2 \pi i} \oint_{\gamma} \frac{1}{z} d z=-1 \quad, \quad \oint_{\gamma} \frac{1}{\bar{z}} d \bar{z}=\overline{\left(\oint_{\gamma} \frac{1}{z} d z\right)},
$$

where $\gamma$ is an integration path in the complex space enclosing the origin.

I.2. Outline of the proof. Next, we outline the key ideas used in the proofs of our main results in Eqs. (I.6), (I.8), and (I.9). For $\vec{P} \in \mathcal{S}, \alpha$ small enough, and $\sigma>0, E_{\vec{P}}^{\sigma}$ is an isolated eigenvalue of $H_{\vec{P}}^{\sigma} \mid \mathcal{F}_{\sigma}$; see Sect. II and Eq. (II.4). Because of the analyticity of $H_{\vec{P}}^{\sigma}$ in the variable $\vec{P}$, it follows that 


$$
\begin{aligned}
& \frac{\partial^{2} E_{|\vec{P}|}^{\sigma}}{(\partial|\vec{P}|)^{2}}=\left.\partial_{i}^{2} E_{|\vec{P}|}^{\sigma}\right|_{\vec{P}=P^{i \hat{i}}} \\
& \quad=1-\left.2\left\langle\frac{1}{2 \pi i} \oint_{\gamma_{\sigma}} \frac{1}{H_{\vec{P}}^{\sigma}-z}\left[\partial_{i} H_{\vec{P}}^{\sigma}\right] \frac{1}{H_{\vec{P}}^{\sigma}-z} d z \Psi_{\vec{P}}^{\sigma},\left[\partial_{i} H_{\vec{P}}^{\sigma}\right] \Psi_{\vec{P}}^{\sigma}\right\rangle\right|_{\vec{P}=P^{i} \hat{i}},
\end{aligned}
$$

where $\partial_{i}=\partial / \partial P^{i}, \hat{i}$ is the unit vector in the direction $i, \Psi_{\vec{P}}^{\sigma}$ is the normalized ground state eigenvector of $H_{\vec{P}}^{\sigma}$ constructed in [6]; $\gamma_{\sigma}$ is a contour path in the complex energy plane enclosing $E_{\vec{P}}^{\sigma}$ and no other point of the spectrum of $\left.H_{\vec{P}}^{\sigma}\right|_{\mathcal{F}_{\sigma}}$, and such that the distance of $\gamma_{\sigma}$ from spec $\left(\left.H_{\vec{P}}^{\sigma}\right|_{\mathcal{F}_{\sigma}}\right)$ is of order $\sigma$.

At first glance, the expression on the R.H. S. of (I.43) might become singular as $\sigma \rightarrow 0$, because the spectral gap above $E_{\vec{P}}^{\sigma}=\inf \operatorname{spec}\left(\left.H_{\vec{P}}^{\sigma}\right|_{\mathcal{F}_{\sigma}}\right)$ is of order $\sigma$. To prove that the limit $\sigma \rightarrow 0$ is, in fact, well defined, we make use of a $\sigma$-dependent Bogoliubov transformation, $W_{\sigma}\left(\vec{\nabla} E_{\vec{P}}^{\sigma}\right)$ (see Sect. II, Eq. (II.3)). This transformation has already been employed in [6] to analyze mass shell properties. In fact, conjugation of $H_{\vec{P}}^{\sigma}$ by $W_{\sigma}\left(\vec{\nabla} E_{\vec{P}}^{\sigma}\right)$ yields an infrared regularized Hamiltonian

$$
K_{\vec{P}}^{\sigma}:=W_{\sigma}\left(\vec{\nabla} E_{\vec{P}}^{\sigma}\right) H_{\vec{P}}^{\sigma} W_{\sigma}^{*}\left(\vec{\nabla} E_{\vec{P}}^{\sigma}\right)
$$

with the property that the corresponding ground state, $\Phi_{\vec{P}}^{\sigma}$, has a non-zero limit, as $\sigma \rightarrow 0$. The Hamiltonian $K_{\vec{P}}^{\sigma}$ has a "canonical form" derived in [6] (see also [12], where a similar operator has been used in the analysis of the Nelson model):

$$
K_{\vec{P}}^{\sigma}=\frac{\left(\vec{\Gamma}_{\vec{P}}^{\sigma}\right)^{2}}{2}+\sum_{\lambda} \int_{\mathbb{R}^{3}}|\vec{k}| \delta_{\vec{P}}^{\sigma}(\hat{k}) b_{\vec{k}, \lambda}^{*} b_{\vec{k}, \lambda} d^{3} k+\mathcal{E}_{\vec{P}}^{\sigma},
$$

where $\delta_{\vec{P}}^{\sigma}(\hat{k})$ is defined in Eq. (II.18), $\mathcal{E}_{\vec{P}}^{\sigma}$ is a c-number defined in Eq. (II.42), and $\vec{\Gamma}_{\vec{P}}^{\sigma}$ is a vector operator defined in Eq. (II.40) starting from Eqs. (II.16), (II.17), (II.34). By construction,

$$
\left\langle\Phi_{\vec{P}}^{\sigma}, \vec{\Gamma}_{\vec{P}}^{\sigma} \Phi_{\vec{P}}^{\sigma}\right\rangle=0
$$

This is a crucial property in the proof of existence of a limit of $\Phi_{\vec{P}}^{\sigma}$ as $\sigma \rightarrow 0$.

Equation (I.46) is also an important ingredient in the proof of (I.6), because, by applying the unitary operator $W_{\sigma}\left(\vec{\nabla} E_{\vec{P}}^{\sigma}\right)$ to each term of the scalar product on the R.H.S. of (I.43) and using (I.46) (see Sect. III), one finds that

$$
\begin{aligned}
\text { (I.43) }= & 1-2 \frac{1}{\left\|\Phi_{\vec{P}}^{\sigma_{j}}\right\|^{2}}\left\langle\frac{1}{2 \pi i} \oint_{\gamma_{j}} \frac{1}{K_{\vec{P}}^{\sigma_{j}}-z_{j}}\left[\partial_{i} E_{\vec{P}}^{\sigma_{j}}-\left(\Gamma_{\vec{P}}^{\sigma_{j}}\right)^{i}\right] \frac{1}{K_{\vec{P}}^{\sigma_{j}}-z_{j}} d z_{j} \Phi_{\vec{P}}^{\sigma_{j}},\right. \\
& {\left.\left[\partial_{i} E_{\vec{P}}^{\sigma_{j}}-\left(\Gamma_{\vec{P}}^{\sigma_{j}}\right)^{i}\right] \Phi_{\vec{P}}^{\sigma_{j}}\right\rangle\left.\right|_{\vec{P}=P^{i \hat{i}}} } \\
= & 1-2\left\langle\frac{1}{2 \pi i} \oint_{\gamma_{\sigma}} \frac{1}{K_{\vec{P}}^{\sigma}-z}\left(\Gamma_{\vec{P}}^{\sigma}\right)^{i} \frac{1}{K_{\vec{P}}^{\sigma}-z} d z \frac{\Phi_{\vec{P}}^{\sigma}}{\left\|\Phi_{\vec{P}}^{\sigma}\right\|},\left.\left(\Gamma_{\vec{P}}^{\sigma}\right)^{i} \frac{\Phi_{\vec{P}}^{\sigma}}{\left\|\Phi_{\vec{P}}^{\sigma}\right\|}\right|_{\vec{P}=P i \hat{i}} .\right.
\end{aligned}
$$


Notice that, if one starts from the two expressions on the R.H.S. of Eq. (I.43) and on the R.H.S. of Eq. (I.47) respectively, both formally expanded in powers of $\alpha^{1 / 2}$, the Bogoliubov transformation can be seen as a tool to re-collect an infinite number of terms and to show a nontrivial identity, thanks only to Eq. (I.46) and to a vanishing contour integration (see Eq. (III.46)). Next, still using Eq. (I.46), one can show that (I.48) remains uniformly bounded in $\sigma$.

To see this we use the inequality

$$
\left|\left\langle\left(\Gamma_{\vec{P}}^{\sigma}\right)^{i} \Phi_{\vec{P}}^{\sigma},\left(\frac{1}{K_{\vec{P}}^{\sigma}-z}\right)^{2}\left(\Gamma_{\vec{P}}^{\sigma}\right)^{i} \Phi_{\vec{P}}^{\sigma}\right\rangle\right| \leq \mathcal{O}\left(\frac{1}{\alpha^{\frac{1}{2}} \sigma^{2 \delta}}\right),
$$

for an arbitrarily small $\delta>0$, with $z \in \gamma_{\sigma}$ and $\alpha$ small enough depending on $\delta$. This inequality will be proven inductively (see Theorem III.1) by introducing sequences of infrared cut-offs $\sigma_{j}$, where $\sigma_{j} \rightarrow 0$ as $j \rightarrow \infty$. The proof by induction is combined with an improved (as compared to the result in [6]) estimate of the rate of convergence of $\left\{\Phi_{\vec{P}}^{\sigma}\right\}$ as $\sigma \rightarrow 0$.

By telescoping, one can plug these improved estimates into (I.48) to end up with the desired uniform bound. The control of the rate of convergence of the R.H.S. in (I.48), as $\sigma \rightarrow 0$, combined with the smoothness in $\vec{P}$, for arbitrary infrared cutoff $\sigma>0$, finally entails the Hölder-continuity in $\vec{P}$ of the limiting quantity

$$
\Sigma_{|\vec{P}|}:=1-\left.\lim _{\sigma \rightarrow 0} 2\left\langle\frac{1}{2 \pi i} \oint_{\gamma_{\sigma}} \frac{1}{K_{\vec{P}}^{\sigma}-z}\left(\Gamma_{\vec{P}}^{\sigma}\right)^{i} \frac{1}{K_{\vec{P}}^{\sigma}-z} d z \frac{\Phi_{\vec{P}}^{\sigma}}{\left\|\Phi_{\vec{P}}^{\sigma}\right\|},\left(\Gamma_{\vec{P}}^{\sigma}\right)^{i} \frac{\Phi_{\vec{P}}^{\sigma}}{\left\|\Phi_{\vec{P}}^{\sigma}\right\|}\right\rangle\right|_{\vec{P}=P^{i} \hat{i}} .
$$

The Hölder-continuity in $\vec{P}$ of $\Sigma_{|\vec{P}|}$ and of $\lim _{\sigma \rightarrow 0} \frac{\partial E_{|\vec{P}|}^{\sigma}}{\partial|\vec{P}|}$, combined with the fundamental theorem of calculus, imply that $E_{\vec{P}}$ is twice differentiable and $\frac{\partial^{2} E_{|\vec{P}|}}{(\partial|\vec{P}|)^{2}} \equiv \Sigma_{|\vec{P}|}$.

Our paper is organized as follows: In Sect. II, we recall how to construct the ground states of the Hamiltonians $H_{\vec{P}}^{\sigma}$ and $K_{\vec{P}}^{\sigma}$ by iterative analytic perturbation theory. This section contains an explicit derivation of the formula of the transformed Hamiltonians and of related algebraic identities that will be used later on.

In Sect. III, we first derive inequality (I.49) and the improved convergence rate of $\left\{\Phi_{\vec{P}}^{\sigma}\right\}$ as $\sigma \rightarrow 0$, by using some key ingredients described in Sect. II. Section III.1 is devoted to an analysis of (I.43) that culminates in the following main results.

Theorem. For $\alpha$ small enough and $\vec{P} \in \mathcal{S}, \frac{\partial^{2} E_{|\vec{P}|}^{\sigma}}{(\partial|\vec{P}|)^{2}}$ converges as $\sigma \rightarrow 0$. The limiting function $\Sigma_{|\vec{P}|}:=\lim _{\sigma \rightarrow 0} \frac{\partial E_{|\vec{P}|}^{\sigma}}{(\partial|\vec{P}|)^{2}}$ is Hölder continuous in $\vec{P} \in \mathcal{S}$. The limit

$$
\lim _{\alpha \rightarrow 0} \Sigma_{|\vec{P}|}=1
$$

holds true uniformly in $\vec{P} \in \mathcal{S}$.

Corollary. For $\alpha$ small enough, the function $E_{\vec{P}}:=\lim _{\sigma \rightarrow 0} E_{\vec{P}}^{\sigma}, \vec{P} \in \mathcal{S}$, is twice differentiable, and

$$
\frac{\partial^{2} E_{|\vec{P}|}}{(\partial|\vec{P}|)^{2}}=\Sigma_{|\vec{P}|} \text { for all } \vec{P} \in \mathcal{S}
$$


Remark. For the complete proof of the construction of the ground states of the Hamiltonians $H_{\vec{P}}^{\sigma}$ and $K_{\vec{P}}^{\sigma}$ by iterative analytic perturbation theory, the reader is advised to consult ref. [6].

\section{Sequences of Ground State Vectors}

In this section, we report on results contained in [6] concerning the ground states of the Hamiltonians $H_{\vec{P}}^{\sigma_{j}}$, where $\vec{P} \in \mathcal{S}$ and $j \in \mathbb{N}_{0}$, and the existence of a limiting vector for the sequence of ground state vectors of the transformed Hamiltonians, $K_{\vec{P}}^{\sigma_{j}}$, where the Bogoliubov transformation used to obtain $K_{\vec{P}}^{\sigma_{j}}$ from $H_{\vec{P}}^{\sigma_{j}}$ (derived in [4]) is determined by

$$
\begin{aligned}
& b_{\vec{k}, \lambda}^{*} \rightarrow W_{\sigma_{j}}\left(\vec{\nabla} E_{\vec{P}}^{\sigma_{j}}\right) b_{\vec{k}, \lambda}^{*} W_{\sigma_{j}}^{*}\left(\vec{\nabla} E_{\vec{P}}^{\sigma_{j}}\right)=b_{\vec{k}, \lambda}^{*}-\alpha^{\frac{1}{2}} \frac{\vec{\nabla} E_{\vec{P}}^{\sigma_{j}} \cdot \vec{\epsilon}_{\vec{k}, \lambda}^{*}}{|\vec{k}|^{\frac{3}{2}} \delta_{\vec{P}}^{\sigma_{j}}(\hat{k})}, \\
& b_{\vec{k}, \lambda} \rightarrow W_{\sigma_{j}}\left(\vec{\nabla} E_{\vec{P}}^{\sigma_{j}}\right) b_{\vec{k}, \lambda} W_{\sigma_{j}}^{*}\left(\vec{\nabla} E_{\vec{P}}^{\sigma_{j}}\right)=b_{\vec{k}, \lambda}-\alpha^{\frac{1}{2}} \frac{\vec{\nabla} E_{\vec{P}}^{\sigma_{j}} \cdot \vec{\epsilon}_{\vec{k}, \lambda}}{|\vec{k}|^{\frac{3}{2}} \delta_{\vec{P}}^{\sigma_{j}}(\hat{k})},
\end{aligned}
$$

for $\vec{k} \in \mathcal{B}_{\Lambda} \backslash \mathcal{B}_{\sigma_{j}}$, with

$$
W_{\sigma_{j}}\left(\vec{\nabla} E_{\vec{P}}^{\sigma_{j}}\right):=\exp \left(\alpha^{\frac{1}{2}} \sum_{\lambda} \int_{\mathcal{B}_{\Lambda} \backslash \mathcal{B}_{\sigma_{j}}} d^{3} k \frac{\vec{\nabla} E_{\vec{P}}^{\sigma_{j}}}{|\vec{k}|^{\frac{3}{2}} \delta_{\vec{P}}^{\sigma_{j}}(\hat{k})} \cdot\left(\vec{\epsilon}_{\vec{k}, \lambda} b_{\vec{k}, \lambda}^{*}-\text { h.c. }\right)\right),
$$

where $\delta_{\vec{P}}^{\sigma_{j}}(\hat{k})$ is defined in (II.18).

II.1. Ground states of the Hamiltonians $H_{\vec{P}}^{\sigma_{j}}$. In [6], the first step consists in constructing the ground states of the regularized fiber Hamiltonians $H_{\vec{P}}^{\sigma_{j}}$. As shown in [6], $H_{\vec{P}}^{\sigma_{j}}$ has a unique ground state, $\Psi_{\vec{P}}^{\sigma_{j}}$, that can be constructed by iterative analytic perturbation theory, as developed in [12]. To recall how this method works some preliminary definitions and results are needed:

- We introduce the Fock spaces

$$
\mathcal{F}_{\sigma_{j}}:=\mathcal{F}^{b}\left(L^{2}\left(\left(\mathbb{R}^{3} \backslash \mathcal{B}_{\sigma_{j}}\right) \times \mathbb{Z}_{2}\right)\right) \quad, \quad \mathcal{F}_{\sigma_{j+1}}^{\sigma_{j}}:=\mathcal{F}^{b}\left(L^{2}\left(\left(\mathcal{B}_{\sigma_{j}} \backslash \mathcal{B}_{\sigma_{j+1}}\right) \times \mathbb{Z}_{2}\right)\right) .
$$

Note that

$$
\mathcal{F}_{\sigma_{j+1}}=\mathcal{F}_{\sigma_{j}} \otimes \mathcal{F}_{\sigma_{j+1}}^{\sigma_{j}}
$$

If not specified otherwise, $\Omega_{f}$ denotes the vacuum state in any one of these Fock spaces. Any vector $\phi$ in $\mathcal{F}_{\sigma_{j}}$ can be identified with the corresponding vector, $\phi \otimes \Omega_{f}$, in $\mathcal{F}$, where $\Omega_{f}$ is the vacuum in $\mathcal{F}_{0}^{\sigma_{j}}$. 


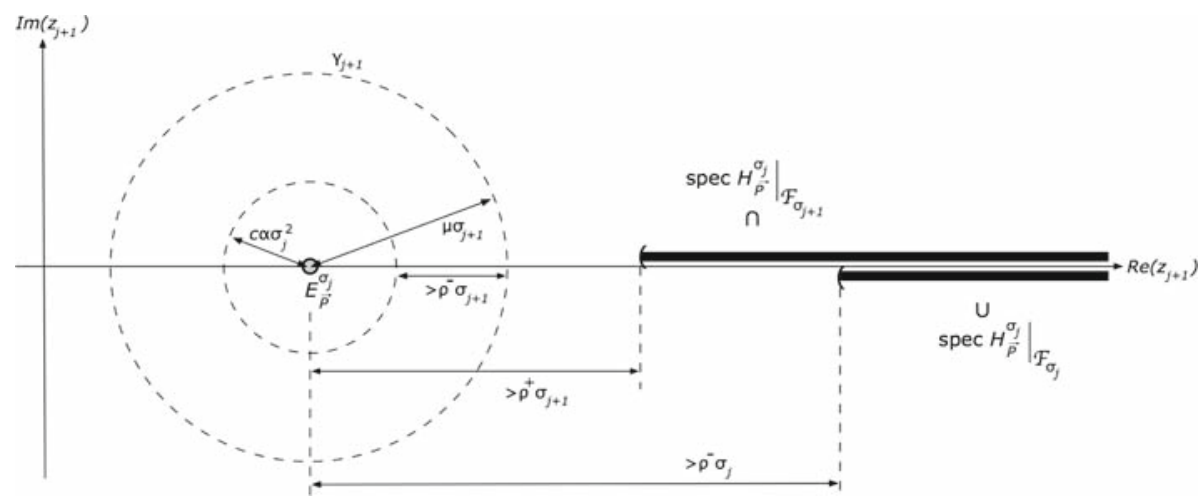

Fig. 1. The contour integral in the energy plane and the gaps

- Momentum-slice interaction Hamiltonians are defined by

$$
\left.\Delta H_{\vec{P}}\right|_{\sigma_{j+1}} ^{\sigma_{j}}:=\left.\alpha^{\frac{1}{2}} \vec{\nabla} \vec{P} H_{\vec{P}}^{\sigma_{j}} \cdot \vec{A}\right|_{\sigma_{j+1}} ^{\sigma_{j}}+\frac{\alpha}{2}\left(\left.\vec{A}\right|_{\sigma_{j+1}} ^{\sigma_{j}}\right)^{2}
$$

where

$$
\left.\vec{A}\right|_{\sigma_{j+1}} ^{\sigma_{j}}:=\sum_{\lambda} \int_{\mathcal{B}_{\sigma_{j}} \backslash \mathcal{B}_{\sigma_{j+1}}} \frac{d^{3} k}{\sqrt{|\vec{k}|}}\left\{\vec{\varepsilon}_{\vec{k}, \lambda} b_{\vec{k}, \lambda}^{*}+\vec{\varepsilon}_{\vec{k}, \lambda}^{*} b_{\vec{k}, \lambda}\right\} .
$$

- Four real parameters, $\epsilon, \rho^{+}, \rho^{-}$, and $\mu$, will appear in our analysis. They have the properties

$$
\begin{aligned}
& 0<\rho^{-}<\mu<\rho^{+}<1-C_{\alpha}<\frac{2}{3}, \\
& 0<\epsilon<\frac{\rho^{-}}{\rho^{+}},
\end{aligned}
$$

where $C_{\alpha}$, with $\frac{1}{3}<C_{\alpha}<1$, for $\alpha$ small enough, is a constant such that the inequality

$$
E_{\vec{P}-\vec{k}}^{\sigma}>E_{\vec{P}}^{\sigma}-C_{\alpha}|\vec{k}|
$$

holds for all $\vec{P} \in \mathcal{S}$ and any $\vec{k} \neq 0$. Here $E_{\vec{P}-\vec{k}}^{\sigma}:=\inf \operatorname{spec} H_{\vec{P}-\vec{k}}^{\sigma}$. We note that $C_{\alpha} \rightarrow \frac{1}{3}$, as $\alpha \rightarrow 0$ (see Statement (I) of Theorem 3.1 in [6]).

By iterative analytic perturbation theory (see [6]), one derives the following results, valid for sufficiently small $\alpha$, depending on our choice of $\Lambda, \epsilon, \rho^{-}, \mu$, and $\rho^{+}$(see also Fig. 1):

(A1) $E_{\vec{P}}^{\sigma_{j}}$ is an isolated simple eigenvalue of $H_{\vec{P}}^{\sigma_{j}} \mid \mathcal{F}_{\sigma_{j}}$ with spectral gap larger or equal to $\rho^{-} \sigma_{j}$. Furthermore, $E_{\vec{P}}^{\sigma_{j}}$ is also the ground state energy of $H_{\vec{P}}^{\sigma_{j}} \mid \mathcal{F}_{\sigma_{j+1}}$, and it is an isolated simple eigenvalue of $H_{\vec{P}}^{\sigma_{j}} \mid \mathcal{F}_{\sigma_{j+1}}$ with spectral gap larger or equal to $\rho^{+} \sigma_{j+1}$. 
$(\mathcal{A} 2)$ The ground-state energies $E_{\vec{P}}^{\sigma_{j}}$ and $E_{\vec{P}}^{\sigma_{j+1}}$ of the Hamiltonians $H_{\vec{P}}^{\sigma_{j}}$ and $H_{\vec{P}}^{\sigma_{j+1}}$, respectively, (acting on the same space $\mathcal{F}_{\sigma_{j+1}}$ ) satisfy the inequalities

$$
0 \leq E_{\vec{P}}^{\sigma_{j+1}} \leq E_{\vec{P}}^{\sigma_{j}}+c \alpha \sigma_{j}^{2},
$$

where $c>0$ is independent of $j$ and of $\alpha$.

(A3) The ground state vectors, $\Psi_{\vec{P}}^{\sigma_{j+1}}$, of $H_{\vec{P}}^{\sigma_{j+1}}$ can be recursively constructed starting from $\Psi_{\vec{P}}^{\sigma_{0}} \equiv \Omega_{f}$ with the help of the spectral projection

$$
\frac{1}{2 \pi i} \oint_{\gamma_{j+1}} d z_{j+1} \frac{1}{H_{\vec{P}}^{\sigma_{j+1}}-z_{j+1}} .
$$

More precisely,

$$
\begin{aligned}
\Psi_{\vec{P}}^{\sigma_{j+1}}:= & \frac{1}{2 \pi i} \oint_{\gamma_{j+1}} d z_{j+1} \frac{1}{H_{\vec{P}}^{\sigma_{j+1}}-z_{j+1}} \Psi_{\vec{P}}^{\sigma_{j}} \\
= & \frac{1}{2 \pi i} \sum_{n=0}^{\infty} \oint_{\gamma_{j+1}} d z_{j+1} \frac{1}{H_{\vec{P}}^{\sigma_{j}}-z_{j+1}} \\
& \times\left[-\left.\Delta H_{\vec{P}}\right|_{\sigma_{j+1}} ^{\sigma_{j}} \frac{1}{H_{\vec{P}}^{\sigma_{j}}-z_{j+1}}\right]^{n} \Psi_{\vec{P}}^{\sigma_{j}},
\end{aligned}
$$

where $\gamma_{j+1}:=\left\{z_{j+1} \in \mathbb{C}|| z_{j+1}-E_{\vec{P}}^{\sigma_{j}} \mid=\mu \sigma_{j+1}\right\}$, with $\mu$ as in (II.8). $\Psi_{\vec{P}}^{\sigma_{j+1}}$ is the (unnormalized) ground state vector of $H_{\vec{P}}^{\sigma_{j+1}} \mid \mathcal{F}_{\sigma}$ for any $0 \leq \sigma \leq \sigma_{j+1}$.

II.2. Transformed Hamiltonians. In this section, we consider the (Bogoliubov-transformed) Hamiltonians

$$
K_{\vec{P}}^{\sigma_{j}}:=W_{\sigma_{j}}\left(\vec{\nabla} E_{\vec{P}}^{\sigma_{j}}\right) H_{\vec{P}}^{\sigma_{j}} W_{\sigma_{j}}^{*}\left(\vec{\nabla} E_{\vec{P}}^{\sigma_{j}}\right)
$$

with ground state vectors $\Phi_{\vec{P}}^{\sigma_{j}}, j=0,1,2,3, \ldots$. Some algebraic manipulations to express $K_{\vec{P}}^{\sigma_{j}}$ in a "canonical form" appear to represent a crucial step before iterative perturbation theory can be applied to the sequence of these transformed Hamiltonians. In addition, some intermediate Hamiltonians, denoted $\hat{K}_{\vec{P}}^{\sigma_{j}}$, must be introduced to arrive at the right kind of convergence estimates.

The same algebraic relations that are used to obtain the "canonical form" of $K_{\vec{P}}^{\sigma_{j}}$ also play an important role in the proof of our main result concerning the limiting behavior, as $\sigma \rightarrow 0$, of the second derivative of the ground state energy $E_{\vec{P}}^{\sigma}$. It is therefore useful to derive the "canonical form" of $K_{\vec{P}}^{\sigma_{j}}$ and the relevant algebraic identities in some detail.

The Feynman-Hellman formula (which holds because $\left(H_{\vec{P}}^{\sigma_{j}}\right)_{\vec{P} \in \mathcal{S}}$ is an analytic family of type A, and $E_{\vec{P}}^{\sigma_{j}}$ is an isolated eigenvalue of $H_{\vec{P}}^{\sigma_{j}} \mid \mathcal{F}_{\sigma}, 0<\sigma \leq \sigma_{j}$ ) yields the identity

$$
\vec{\nabla} E_{\vec{P}}^{\sigma_{j}}=\vec{P}-\left\langle\vec{P}^{f}-\alpha^{\frac{1}{2}} \vec{A}^{\sigma_{j}}\right\rangle_{\psi_{\vec{P}}^{\sigma_{j}}}
$$


where, given an operator $B$ and a vector $\psi$ in the domain of $B$, we use the notation

$$
\langle B\rangle_{\psi}:=\frac{\langle\psi, B \psi\rangle}{\langle\psi, \psi\rangle} .
$$

As stated in [6], for $\alpha$ small enough,

$$
\sup _{\vec{P} \in \mathcal{S}}\left|\vec{\nabla} E_{\vec{P}}^{\sigma_{j}}\right|<1 \quad \forall j \in \mathbb{N}_{0}
$$

We define

$$
\begin{aligned}
\vec{\beta}^{\sigma_{j}} & :=\vec{P}^{f}-\alpha^{\frac{1}{2}} \vec{A}^{\sigma_{j}}, \\
\delta_{\vec{P}}^{\sigma_{j}}(\hat{k}) & :=1-\hat{k} \cdot \vec{\nabla} E_{\vec{P}}^{\sigma_{j}}, \quad \hat{k}:=\frac{\vec{k}}{|\vec{k}|}, \quad\left|\vec{\nabla} E_{\vec{P}}^{\sigma_{j}}\right|<1, \\
c_{\vec{k}, \lambda}^{*} & :=b_{\vec{k}, \lambda}^{*}+\alpha^{\frac{1}{2}} \frac{\vec{\nabla} E_{\vec{P}}^{\sigma_{j}} \cdot \vec{\epsilon}_{\vec{k}, \lambda}^{*}}{|\vec{k}|^{\frac{3}{2}} \delta_{\vec{P}}^{\sigma_{j}}(\hat{k})}, \\
c_{\vec{k}, \lambda} & :=b_{\vec{k}, \lambda}+\alpha^{\frac{1}{2}} \frac{\vec{\nabla} E_{\vec{P}}^{\sigma_{j}} \cdot \vec{\epsilon}_{\vec{k}, \lambda}}{|\vec{k}|^{\frac{3}{2}} \delta_{\vec{P}}^{\sigma_{j}}(\hat{k})} .
\end{aligned}
$$

We rewrite $H_{\vec{P}}^{\sigma_{j}}$ as

$$
H_{\vec{P}}^{\sigma_{j}}=\frac{\left(\vec{P}-\vec{\beta}^{\sigma_{j}}\right)^{2}}{2}+H^{f},
$$

and, using (II.15) and (II.17),

$$
\vec{P}=\vec{\nabla} E_{\vec{P}}^{\sigma_{j}}+\left\langle\vec{\beta}^{\sigma_{j}}\right\rangle_{\psi_{\vec{P}}^{\sigma_{j}}}
$$

We then obtain

$$
\begin{aligned}
H_{\vec{P}}^{\sigma_{j}}= & \frac{\vec{P}^{2}}{2}-\left(\vec{\nabla} E_{\vec{P}}^{\sigma_{j}}+\left\langle\vec{\beta}^{\sigma_{j}}\right\rangle_{\psi_{\vec{P}}^{\sigma_{j}}}\right) \cdot \vec{\beta}^{\sigma_{j}}+\frac{\vec{\beta}^{\sigma_{j}^{2}}}{2}+H^{f} \\
= & \frac{\vec{P}^{2}}{2}+\frac{\vec{\beta}^{\sigma_{j}^{2}}}{2}-\left\langle\vec{\beta}^{\sigma_{j}}\right\rangle_{\psi_{\vec{P}}^{\sigma_{j}}} \cdot \vec{\beta}^{\sigma_{j}} \\
& +\sum_{\lambda} \int_{\mathbb{R}^{3} \backslash\left(\mathcal{B}_{\Lambda} \backslash \mathcal{B}_{\sigma_{j}}\right)}|\vec{k}| \delta_{\vec{P}}^{\sigma_{j}}(\hat{k}) b_{\vec{k}, \lambda}^{*} b_{\vec{k}, \lambda} d^{3} k \\
& +\sum_{\lambda} \int_{\mathcal{B}_{\Lambda} \backslash \mathcal{B}_{\sigma_{j}}}|\vec{k}| \delta_{\vec{P}}^{\sigma_{j}}(\hat{k}) c_{\vec{k}, \lambda}^{*} c_{\vec{k}, \lambda} d^{3} k \\
& -\alpha \sum_{\lambda} \int_{\mathcal{B}_{\Lambda} \backslash \mathcal{B}_{\sigma_{j}}}|\vec{k}| \delta_{\vec{P}}^{\sigma_{j}}(\hat{k}) \frac{\vec{\nabla} E_{\vec{P}}^{\sigma_{j}} \cdot \vec{\epsilon}_{\vec{k}, \lambda}}{|\vec{k}|^{\frac{3}{2}} \delta_{\vec{P}}^{\sigma_{j}}(\hat{k})} \frac{\left.\vec{\nabla} E_{\vec{P}}^{\sigma_{j}} \cdot \overrightarrow{\epsilon_{\vec{k}}}\right|^{\frac{3}{2}} \delta_{\vec{P}}^{\sigma_{j}}(\hat{k})}{d^{3} k} .
\end{aligned}
$$


Adding and subtracting $1 / 2\left\langle\vec{\beta}^{\sigma_{j}}\right\rangle_{\psi_{\vec{P}}^{\sigma_{j}}}^{2}$, one finds that

$$
\begin{aligned}
& H_{\vec{P}}^{\sigma_{j}}=\frac{\vec{P}^{2}}{2}-\frac{\left\langle\vec{\beta}^{\sigma_{j}}\right\rangle_{\psi_{\vec{P}}^{\sigma_{j}}}^{2}}{2}+\frac{\left(\vec{\beta}^{\sigma_{j}}-\left\langle\vec{\beta}^{\sigma_{j}}\right\rangle_{\psi_{\vec{P}}^{\sigma_{j}}}\right)^{2}}{2} \\
& +\sum_{\lambda} \int_{\mathbb{R}^{3} \backslash\left(\mathcal{B}_{\Lambda} \backslash \mathcal{B}_{\sigma_{j}}\right)}|\vec{k}| \delta_{\vec{P}}^{\sigma_{j}}(\hat{k}) b_{\vec{k}, \lambda}^{*} b_{\vec{k}, \lambda} d^{3} k \\
& +\sum_{\lambda} \int_{\mathcal{B}_{\Lambda} \backslash \mathcal{B}_{\sigma_{j}}}|\vec{k}| \delta_{\vec{P}}^{\sigma_{j}}(\hat{k}) c_{\vec{k}, \lambda}^{*} c_{\vec{k}, \lambda} d^{3} k \\
& -\alpha \sum_{\lambda} \int_{\mathcal{B}_{\Lambda} \backslash \mathcal{B}_{\sigma_{j}}}|\vec{k}| \delta_{\vec{P}}^{\sigma_{j}}(\hat{k}) \frac{\vec{\nabla} E_{\vec{P}}^{\sigma_{j}} \cdot \vec{\epsilon}_{\vec{k}, \lambda}}{|\vec{k}|^{\frac{3}{2}} \delta_{\vec{P}}^{\sigma_{j}}(\hat{k})} \frac{\vec{\nabla} E_{\vec{P}}^{\sigma_{j}} \cdot \vec{\epsilon}_{\vec{k}, \lambda}^{*}}{|\vec{k}|^{\frac{3}{2}} \delta_{\vec{P}}^{\sigma_{j}}(\hat{k})} d^{3} k .
\end{aligned}
$$

Next, we implement the Bogoliubov transformation

$$
\begin{aligned}
& b_{\vec{k}, \lambda}^{*} \rightarrow W_{\sigma_{j}}\left(\vec{\nabla} E_{\vec{P}}^{\sigma_{j}}\right) b_{\vec{k}, \lambda}^{*} W_{\sigma_{j}}^{*}\left(\vec{\nabla} E_{\vec{P}}^{\sigma_{j}}\right)=b_{\vec{k}, \lambda}^{*}-\alpha^{\frac{1}{2}} \frac{\vec{\nabla} E_{\vec{P}}^{\sigma_{j}} \cdot \vec{\epsilon}_{\vec{k}, \lambda}^{*}}{|\vec{k}|^{\frac{3}{2}} \delta_{\vec{P}}^{\sigma_{j}}(\hat{k})}, \\
& b_{\vec{k}, \lambda} \rightarrow W_{\sigma_{j}}\left(\vec{\nabla} E_{\vec{P}}^{\sigma_{j}}\right) b_{\vec{k}, \lambda} W_{\sigma_{j}}^{*}\left(\vec{\nabla} E_{\vec{P}}^{\sigma_{j}}\right)=b_{\vec{k}, \lambda}-\alpha^{\frac{1}{2}} \frac{\vec{\nabla} E_{\vec{P}}^{\sigma_{j}} \cdot \vec{\epsilon}_{\vec{k}, \lambda}}{|\vec{k}|^{\frac{3}{2}} \delta_{\vec{P}}^{\sigma_{j}}(\hat{k})},
\end{aligned}
$$

for $\vec{k} \in \mathcal{B}_{\Lambda} \backslash \mathcal{B}_{\sigma_{j}}$, where $W_{\sigma_{j}}\left(\vec{\nabla} E_{\vec{P}}^{\sigma_{j}}\right)$ is defined in (II.3). It is evident that $W_{\sigma_{j}}$ acts as the identity on $\mathcal{F}^{b}\left(L^{2}\left(\mathcal{B}_{\sigma_{j}} \times \mathbb{Z}_{2}\right)\right)$ and on $\mathcal{F}^{b}\left(L^{2}\left(\left(\mathbb{R}^{3} \backslash \mathcal{B}_{\Lambda}\right) \times \mathbb{Z}_{2}\right)\right)$.

We define the vector operators

$$
\begin{aligned}
\vec{\Pi}_{\vec{P}}^{\sigma_{j}}:= & W_{\sigma_{j}}\left(\vec{\nabla} E_{\vec{P}}^{\sigma_{j}}\right) \vec{\beta}^{\sigma_{j}} W_{\sigma_{j}}^{*}\left(\vec{\nabla} E_{\vec{P}}^{\sigma_{j}}\right) \\
& -\left\langle W_{\sigma_{j}}\left(\vec{\nabla} E_{\vec{P}}^{\sigma_{j}}\right) \vec{\beta}^{\sigma_{j}} W_{\sigma_{j}}^{*}\left(\vec{\nabla} E_{\vec{P}}^{\sigma_{j}}\right)\right\rangle_{\Omega_{f}},
\end{aligned}
$$

noting that, by (II.15), (II.17), and (II.34),

$$
\begin{aligned}
\left\langle\vec{\beta}^{\sigma_{j}}\right\rangle_{\psi_{\vec{P}}}^{\sigma_{j}} & =\vec{P}-\vec{\nabla} E_{\vec{P}}^{\sigma_{j}} \\
& =\frac{\left\langle\Phi_{\vec{P}}^{\sigma_{j}}, \vec{\Pi}_{\vec{P}}^{\sigma_{j}} \Phi_{\vec{P}}^{\sigma_{j}}\right\rangle}{\left\langle\Phi_{\vec{P}}^{\sigma_{j}}, \Phi_{\vec{P}}^{\sigma_{j}}\right\rangle}+\left\langle W_{\sigma_{j}}\left(\vec{\nabla} E_{\vec{P}}^{\sigma_{j}}\right) \vec{\beta}^{\sigma_{j}} W_{\sigma_{j}}^{*}\left(\vec{\nabla} E_{\vec{P}}^{\sigma_{j}}\right)\right\rangle_{\Omega_{f}},
\end{aligned}
$$

where $\Phi_{\vec{P}}^{\sigma_{j}}$ is the ground state of the Bogoliubov-transformed Hamiltonian

$$
K_{\vec{P}}^{\sigma_{j}}:=W_{\sigma_{j}}\left(\vec{\nabla} E_{\vec{P}}^{\sigma_{j}}\right) H_{\vec{P}}^{\sigma_{j}} W_{\sigma_{j}}^{*}\left(\vec{\nabla} E_{\vec{P}}^{\sigma_{j}}\right) .
$$

Notice that in (II.36) only the ray of $\Phi_{\vec{P}}^{\sigma_{j}}$ enters. The sequence of vectors $\left\{\Phi_{\vec{P}}^{\sigma_{j}}\right\}$ is defined in Section II.3.

It is easy to see that

$$
W_{\sigma_{j}}\left(\vec{\nabla} E_{\vec{P}}^{\sigma_{j}}\right) \vec{\beta}^{\sigma_{j}} W_{\sigma_{j}}^{*}\left(\vec{\nabla} E_{\vec{P}}^{\sigma_{j}}\right)-\left\langle\vec{\beta}^{\sigma_{j}}\right\rangle_{\Psi_{\vec{P}}^{\sigma_{j}}}=\vec{\Pi}_{\vec{P}}^{\sigma_{j}}-\left\langle\vec{\Pi}_{\vec{P}}^{\sigma_{j}}\right\rangle_{\Phi_{\vec{P}}^{\sigma_{j}}} .
$$


After inspecting straightforward operator domain questions (see [6]), the "canonical form" of $K_{\vec{P}}^{\sigma_{j}}$ is given by

$$
K_{\vec{P}}^{\sigma_{j}}=\frac{\left(\vec{\Gamma}_{\vec{P}}^{\sigma_{j}}\right)^{2}}{2}+\sum_{\lambda} \int_{\mathbb{R}^{3}}|\vec{k}| \delta_{\vec{P}}^{\sigma_{j}}(\hat{k}) b_{\vec{k}, \lambda}^{*} b_{\vec{k}, \lambda} d^{3} k+\mathcal{E}_{\vec{P}}^{\sigma_{j}}
$$

where

$$
\vec{\Gamma}_{\vec{P}}^{\sigma_{j}}:=\vec{\Pi}_{\vec{P}}^{\sigma_{j}}-\left\langle\vec{\Pi}_{\vec{P}}^{\sigma_{j}}\right\rangle_{\Phi}^{\sigma_{\vec{P}}}
$$

so that

$$
\left\langle\vec{\Gamma}_{\vec{P}}^{\sigma_{j}}\right\rangle_{\Phi_{\vec{P}}^{\sigma_{j}}}=0
$$

and

$$
\begin{aligned}
\mathcal{E}_{\vec{P}}^{\sigma_{j}}:= & \frac{\vec{P}^{2}}{2}-\frac{\left(\vec{P}-\vec{\nabla} E_{\vec{P}}^{\sigma_{j}}\right)^{2}}{2} \\
& -\alpha \sum_{\lambda} \int_{\mathcal{B}_{\Lambda} \backslash \mathcal{B}_{\sigma_{j}}}|\vec{k}| \delta_{\vec{P}}^{\sigma_{j}}(\hat{k}) \frac{\vec{\nabla} E_{\vec{P}}^{\sigma_{j}} \cdot \vec{\epsilon}_{\vec{k}, \lambda}}{|\vec{k}|^{\frac{3}{2}} \delta_{\vec{P}}^{\sigma_{j}}(\hat{k})} \frac{\vec{\nabla} E_{\vec{P}}^{\sigma_{j}} \cdot \vec{\epsilon}_{\vec{k}, \lambda}^{*}}{|\vec{k}|^{\frac{3}{2}} \delta_{\vec{P}}^{\sigma_{j}}(\hat{k})} d^{3} k .
\end{aligned}
$$

Equation (II.39) follows by using that

$$
\begin{aligned}
& W_{\sigma_{j}}\left(\vec{\nabla} E_{\vec{P}}^{\sigma_{j}}\right) c_{\vec{k}, \lambda}^{*} W_{\sigma_{j}}^{*}\left(\vec{\nabla} E_{\vec{P}}^{\sigma_{j}}\right)=b_{\vec{k}, \lambda}^{*}, \\
& W_{\sigma_{j}}\left(\vec{\nabla} E_{\vec{P}}^{\sigma_{j}}\right) c_{\vec{k}, \lambda} W_{\sigma_{j}}^{*}\left(\vec{\nabla} E_{\vec{P}}^{\sigma_{j}}\right)=b_{\vec{k}, \lambda},
\end{aligned}
$$

for $\vec{k} \in \mathcal{B}_{\Lambda} \backslash \mathcal{B}_{\sigma_{j}}$.

An intermediate Hamiltonian, $\hat{K}_{\vec{P}}^{\sigma_{j+1}}$, is defined by

$$
\hat{K}_{\vec{P}}^{\sigma_{j+1}}:=W_{\sigma_{j+1}}\left(\vec{\nabla} E_{\vec{P}}^{\sigma_{j}}\right) H_{\vec{P}}^{\sigma_{j+1}} W_{\sigma_{j+1}}^{*}\left(\vec{\nabla} E_{\vec{P}}^{\sigma_{j}}\right),
$$

where

$$
W_{\sigma_{j+1}}\left(\vec{\nabla} E_{\vec{P}}^{\sigma_{j}}\right):=\exp \left(\alpha^{\frac{1}{2}} \sum_{\lambda} \int_{\mathcal{B}_{\Lambda} \backslash \mathcal{B}_{\sigma_{j+1}}} d^{3} k \frac{\vec{\nabla} E_{\vec{P}}^{\sigma_{j}}}{|\vec{k}|^{\frac{3}{2}} \delta_{\vec{P}}^{\sigma_{j}}(\hat{k})} \cdot\left(\vec{\epsilon}_{\vec{k}, \lambda} b_{\vec{k}, \lambda}^{*}-\text { h.c. }\right)\right) .
$$

We decompose $\hat{K}_{\vec{P}}^{\sigma_{j+1}}$ into several different terms, similarly as $K_{\vec{P}}^{\sigma_{j}}$. We recall that

$$
H_{\vec{P}}^{\sigma_{j+1}}=\frac{\left(\vec{P}-\vec{\beta}^{\sigma_{j+1}}\right)^{2}}{2}+H^{f}
$$

and, by (II.35),

$$
\vec{P}=\vec{\nabla} E_{\vec{P}}^{\sigma_{j}}+\left\langle\vec{\beta}^{\sigma_{j}}\right\rangle_{\psi_{\vec{P}}^{\sigma_{j}}}
$$


It follows that (see also (II.28)-(II.31))

$$
\begin{aligned}
H_{\vec{P}}^{\sigma_{j+1}}= & \frac{\vec{P}^{2}}{2}-\left(\vec{\nabla} E_{\vec{P}}^{\sigma_{j}}+\left\langle\vec{\beta}^{\sigma_{j}}\right\rangle_{\psi_{\vec{P}}^{\sigma_{j}}}\right) \cdot \vec{\beta}^{\sigma_{j+1}}+\frac{\vec{\beta}^{\sigma_{j+1}}}{2}+H^{f} \\
= & \frac{\vec{P}^{2}}{2}+\frac{\vec{\beta}^{\sigma_{j+1}^{2}}}{2}-\left\langle\vec{\beta}^{\sigma_{j}}\right\rangle_{\psi_{\vec{P}}^{\sigma_{j}}} \cdot \vec{\beta}^{\sigma_{j+1}} \\
& +\sum_{\lambda} \int_{\mathbb{R}^{3} \backslash\left(\mathcal{B}_{\Lambda} \backslash \mathcal{B}_{\sigma_{j+1}}\right)}|\vec{k}| \delta_{\vec{P}}^{\sigma_{j}}(\hat{k}) b_{\vec{k}, \lambda}^{*} b_{\vec{k}, \lambda} d^{3} k \\
& +\sum_{\lambda} \int_{\mathcal{B}_{\Lambda} \backslash \mathcal{B}_{\sigma_{j+1}}|\vec{k}| \delta_{\vec{P}}^{\sigma_{j}}(\hat{k}) c_{\vec{k}, \lambda}^{*} c_{\vec{k}, \lambda} d^{3} k} \\
& -\alpha \sum_{\lambda} \int_{\mathcal{B}_{\Lambda} \backslash \mathcal{B}_{\sigma_{j+1}}}|\vec{k}| \delta_{\vec{P}}^{\sigma_{j}}(\hat{k}) \frac{\vec{\nabla} E_{\vec{P}}^{\sigma_{j}} \cdot \vec{\epsilon}_{\vec{k}, \lambda}}{|\vec{k}|^{\frac{3}{2}} \delta_{\vec{P}}^{\sigma_{j}}(\hat{k})} \frac{\vec{\nabla} E_{\vec{P}}^{\sigma_{j}} \cdot \vec{\epsilon}_{\vec{k}, \lambda}^{*}}{|\vec{k}|^{\frac{3}{2}} \delta_{\vec{P}}^{\sigma_{j}}(\hat{k})} d^{3} k .
\end{aligned}
$$

We now add and subtract $1 / 2\left\langle\vec{\beta}^{\sigma_{j}}\right\rangle_{\psi_{\vec{P}}^{\sigma_{j}}}^{2}$ and conjugate the resulting operator with the unitary operator $W_{\sigma_{j+1}}\left(\vec{\nabla} E_{\vec{P}}^{\sigma_{j}}\right)$. After inspecting straightforward operator domain questions (see [6]), we find that

$$
\begin{aligned}
\hat{K}_{\vec{P}}^{\sigma_{j+1}}= & \frac{\left(\vec{\Gamma}_{\vec{P}}^{\sigma_{j}}+\overrightarrow{\mathcal{L}}_{\sigma_{j+1}}^{\sigma_{j}}+\overrightarrow{\mathcal{I}}_{\sigma_{j+1}}^{\sigma_{j}}\right)^{2}}{2} \\
& +\sum_{\lambda} \int_{\mathbb{R}^{3}}|\vec{k}| \delta_{\vec{P}}^{\sigma_{j}}(\hat{k}) b_{\vec{k}, \lambda}^{*} b_{\vec{k}, \lambda} d^{3} k+\hat{\mathcal{E}}_{\vec{P}}^{\sigma_{j+1}},
\end{aligned}
$$

where

$$
\begin{aligned}
& \overrightarrow{\mathcal{L}}_{\sigma_{j+1}}^{\sigma_{j}}:=-\alpha^{\frac{1}{2}} \sum_{\lambda} \int_{\mathcal{B}_{\sigma_{j}} \backslash \mathcal{B}_{\sigma_{j+1}}} \vec{k} \frac{\vec{\nabla} E_{\vec{P}}^{\sigma_{j}} \cdot \vec{\epsilon}_{\vec{k}, \lambda}^{*} b_{\vec{k}, \lambda}+\text { h.c. }}{|\vec{k}|^{\frac{3}{2}} \delta_{\vec{P}}^{\sigma_{j}}(\hat{k})} d^{3} k, \\
& -\left.\alpha^{\frac{1}{2}} \vec{A}\right|_{\sigma_{j+1}} ^{\sigma_{j}} \\
& \overrightarrow{\mathcal{I}}_{\sigma_{j+1}}^{\sigma_{j}}:=\alpha \sum_{\lambda} \int_{\mathcal{B}_{\sigma_{j}} \backslash \mathcal{B}_{\sigma_{j+1}}} \vec{k} \frac{\vec{\nabla} E_{\vec{P}}^{\sigma_{j}} \cdot \vec{\epsilon}_{\vec{k}, \lambda}}{|\vec{k}|^{\frac{3}{2}} \delta_{\vec{P}}^{\sigma_{j}}(\hat{k})} \frac{\vec{\nabla} E_{\vec{P}}^{\sigma_{j}} \cdot \vec{\epsilon}_{\vec{k}, \lambda}^{*}}{|\vec{k}|^{\frac{3}{2}} \delta_{\vec{P}}^{\sigma_{j}}(\hat{k})} d^{3} k \\
& +\alpha \sum_{\lambda} \int_{\mathcal{B}_{\sigma_{j}} \backslash \mathcal{B}_{\sigma_{j+1}}}\left[\vec{\epsilon}_{\vec{k}, \lambda} \frac{\vec{\nabla} E_{\vec{P}}^{\sigma_{j}} \cdot \vec{\epsilon}_{\vec{k}, \lambda}^{*}}{|\vec{k}|^{\frac{3}{2}} \delta_{\vec{P}}^{\sigma_{j}}(\hat{k})}+\text { h.c. }\right] \frac{d^{3} k}{\sqrt{|\vec{k}|}}, \\
& \hat{\mathcal{E}}_{\vec{P}}^{\sigma_{\vec{P}+1}}:=\frac{\vec{P}^{2}}{2}-\frac{\left(\vec{P}-\vec{\nabla} E_{\vec{P}}^{\sigma_{j}}\right)^{2}}{2} \\
& -\alpha \sum_{\lambda} \int_{\mathcal{B}_{\Lambda} \backslash \mathcal{B}_{\sigma_{j+1}}}|\vec{k}| \delta_{\vec{P}}^{\sigma_{j}}(\hat{k}) \frac{\vec{\nabla} E_{\vec{P}}^{\sigma_{j}} \cdot \vec{\epsilon}_{\vec{k}, \lambda}}{|\vec{k}|^{\frac{3}{2}} \delta_{\vec{P}}^{\sigma_{j}}(\hat{k})} \frac{\vec{\nabla} E_{\vec{P}}^{\sigma_{j}} \cdot \vec{\epsilon}_{\vec{k}, \lambda}^{*}}{|\vec{k}|^{\frac{3}{2}} \delta_{\vec{P}}^{\sigma_{j}}(\hat{k})} d^{3} k .
\end{aligned}
$$

We also define the operators

$$
\hat{\vec{\Pi}}_{\vec{P}}^{\sigma_{j}}:=W_{\sigma_{j}}\left(\vec{\nabla} E_{\vec{P}}^{\sigma_{j-1}}\right) W_{\sigma_{j}}^{*}\left(\vec{\nabla} E_{\vec{P}}^{\sigma_{j}}\right) \vec{\Pi}_{\vec{P}}^{\sigma_{j}} W_{\sigma_{j}}\left(\vec{\nabla} E_{\vec{P}}^{\sigma_{j}}\right) W_{\sigma_{j}}^{*}\left(\vec{\nabla} E_{\vec{P}}^{\sigma_{j-1}}\right),
$$


and

$$
\hat{\vec{\Gamma}}_{\vec{P}}^{\sigma_{j}}:=\hat{\vec{\Pi}}_{\vec{P}}^{\sigma_{j}}-\left\langle\hat{\vec{\Pi}}_{\vec{P}}^{\sigma_{j}}\right\rangle_{\hat{\Phi}_{\vec{P}}^{\sigma_{j}}}
$$

which are used in the proofs of convergence of the ground state vectors. Here, $\hat{\Phi}_{\vec{P}}^{\sigma_{j}}$ denotes the ground state vector of the Hamiltonian

$$
\hat{K}_{\vec{P}}^{\sigma_{j}}:=W_{\sigma_{j}}\left(\vec{\nabla} E_{\vec{P}}^{\sigma_{j-1}}\right) W_{\sigma_{j}}^{*}\left(\vec{\nabla} E_{\vec{P}}^{\sigma_{j}}\right) K_{\vec{P}}^{\sigma_{j}} W_{\sigma_{j}}\left(\vec{\nabla} E_{\vec{P}}^{\sigma_{j}}\right) W_{\sigma_{j}}^{*}\left(\vec{\nabla} E_{\vec{P}}^{\sigma_{j-1}}\right) .
$$

(The sequence of vectors $\left\{\hat{\Phi}_{\vec{P}}^{\sigma_{j}}\right\}$ is defined in (II.64).)

Notice that

$$
\hat{\vec{\Gamma}}_{\vec{P}}^{\sigma_{j}}=W_{\sigma_{j}}\left(\vec{\nabla} E_{\vec{P}}^{\sigma_{j-1}}\right) W_{\sigma_{j}}^{*}\left(\vec{\nabla} E_{\vec{P}}^{\sigma_{j}}\right) \vec{\Gamma}_{\vec{P}}^{\sigma_{j}} W_{\sigma_{j}}\left(\vec{\nabla} E_{\vec{P}}^{\sigma_{j}}\right) W_{\sigma_{j}}^{*}\left(\vec{\nabla} E_{\vec{P}}^{\sigma_{j-1}}\right)
$$

An important identity used in [6] and in the sequel of the present paper is $(j \geq 1)$

$$
\begin{aligned}
\hat{\vec{\Gamma}}_{\vec{P}}^{\sigma_{j}}-\vec{\Gamma}_{\vec{P}}^{\sigma_{j-1}}= & \vec{\nabla} E_{\vec{P}}^{\sigma_{j}}-\vec{\nabla} E_{\vec{P}}^{\sigma_{j-1}}+\overrightarrow{\mathcal{L}}_{\sigma_{j}}^{\sigma_{j-1}} \\
& +\alpha \sum_{\lambda} \int_{\mathcal{B}_{\sigma_{j-1}} \backslash \mathcal{B}_{\sigma_{j}}} \overrightarrow{\vec{\nabla}} \frac{\vec{\nabla} E_{\vec{P}}^{\sigma_{j-1}} \cdot \vec{\epsilon}_{\vec{k}, \lambda}}{|\vec{k}|^{\frac{3}{2}} \delta_{\vec{P}}^{\sigma_{j-1}}(\hat{k})} \frac{\overrightarrow{\vec{p}} E_{\vec{P}}^{\sigma_{j-1}} \cdot \vec{\epsilon}_{\vec{k}, \lambda}^{*}}{|\vec{k}|^{\frac{3}{2}} \delta_{\vec{P}}^{\sigma_{j-1}}(\hat{k})} d^{3} k \\
& +\alpha \sum_{\lambda} \int_{\mathcal{B}_{\sigma_{j-1}} \backslash \mathcal{B}_{\sigma_{j}}}\left[\overrightarrow{\epsilon_{\vec{k}, \lambda}} \frac{\vec{\nabla} E_{\vec{P}}^{\sigma_{j-1}} \cdot \vec{\epsilon}_{\vec{k}, \lambda}^{*}}{|\vec{k}|^{\frac{3}{2}} \delta_{\vec{P}}^{\sigma_{j-1}}(\hat{k})}+\text { h.c. }\right] \frac{d^{3} k}{\sqrt{|\vec{k}|}} .
\end{aligned}
$$

Equation (II.63) can be derived using (II.34), (II.36), (II.38), (II.40), (II.60), and (II.61).

II.3. Convergence of the sequence $\left\{\Phi_{\vec{P}}^{\sigma_{j}}\right\}_{j=0}^{\infty}$. We start from $\Phi_{\vec{P}}^{\sigma_{0}} \equiv \Omega_{f}$. To pass from momentum scale $j$ to $j+1$, we proceed in two steps: First, we construct an intermediate vector, $\hat{\Phi}_{\vec{P}}^{\sigma_{j+1}}$, defined by

$$
\hat{\Phi}_{\vec{P}}^{\sigma_{j+1}}:=\sum_{n=0}^{\infty} \frac{1}{2 \pi i} \oint_{\gamma_{j+1}} d z_{j+1} \frac{1}{K_{\vec{P}}^{\sigma_{j}}-z_{j+1}}\left[-\left.\Delta K_{\vec{P}}\right|_{\sigma_{j+1}} ^{\sigma_{j}} \frac{1}{K_{\vec{P}}^{\sigma_{j}}-z_{j+1}}\right]^{n} \Phi_{\vec{P}}^{\sigma_{j}},
$$

where

$$
\begin{aligned}
\left.\Delta K_{\vec{P}}\right|_{\sigma_{j+1}} ^{\sigma_{j}} & :=\hat{K}_{\vec{P}}^{\sigma_{j+1}}-\hat{\mathcal{E}}_{\vec{P}}^{\sigma_{j+1}}+\mathcal{E}_{\vec{P}}^{\sigma_{j}}-K_{\vec{P}}^{\sigma_{j}} \\
& =\frac{1}{2}\left(\vec{\Gamma}_{\vec{P}}^{\sigma_{j}} \cdot\left(\overrightarrow{\mathcal{L}}_{\sigma_{j+1}}^{\sigma_{j}}+\overrightarrow{\mathcal{I}}_{\sigma_{j+1}}^{\sigma_{j}}\right)+\text { h.c. }\right)+\frac{1}{2}\left(\overrightarrow{\mathcal{L}}_{\sigma_{j+1}}^{\sigma_{j}}+\overrightarrow{\mathcal{I}}_{\sigma_{j+1}}^{\sigma_{j}}\right)^{2} .
\end{aligned}
$$

Subsequently, we construct $\Phi_{\vec{P}}^{\sigma_{j+1}}$ by setting

$$
\Phi_{\vec{P}}^{\sigma_{j+1}}:=W_{\sigma_{j+1}}\left(\vec{\nabla} E_{\vec{P}}^{\sigma_{j+1}}\right) W_{\sigma_{j+1}}^{*}\left(\vec{\nabla} E_{\vec{P}}^{\sigma_{j}}\right) \hat{\Phi}_{\vec{P}}^{\sigma_{j+1}} .
$$

The series in (II.64) is term-wise well-defined and converges strongly to a non-zero vector, provided $\alpha$ is small enough (independently of $j$ ). The proof of this claim is based on operator-norm estimates of the type used in controlling the Neumann expansion in (II.13), which requires an estimate of the spectral gap that follows from the unitarity of $W_{\sigma_{j}}\left(\vec{\nabla} E_{\vec{P}}^{\sigma_{j}}\right)$ and Result (A1) described after Eq. (II.10). 

term

A key point in our proof of convergence of the sequence $\left\{\Phi_{\vec{P}}^{\sigma_{j}}\right\}$ is to show that the

$$
\vec{\Gamma}_{\vec{P}}^{\sigma_{j}} \cdot\left(\overrightarrow{\mathcal{L}}_{\sigma_{j+1}}^{\sigma_{j}}+\overrightarrow{\mathcal{I}}_{\sigma_{j+1}}^{\sigma_{j}}\right)+\text { h.c. }
$$

appearing in (II.66), which is superficially "marginal" in the infrared, by power counting, is in fact "irrelevant" (using the terminology of renormalization group theory). This is a consequence of the orthogonality condition

$$
\left\langle\Phi_{\vec{P}}^{\sigma_{j}}, \vec{\Gamma}_{\vec{P}}^{\sigma_{j}} \Phi_{\vec{P}}^{\sigma_{j}}\right\rangle=0
$$

which, when combined with an inductive argument, implies that

$$
\left\|\left(\frac{1}{K_{\vec{P}}^{\sigma_{j}}-z_{j+1}}\right)^{\frac{1}{2}}\left[\vec{\Gamma}_{\vec{P}}^{\sigma_{j}} \cdot\left(\overrightarrow{\mathcal{L}}_{\sigma_{j+1}}^{\sigma_{j}(+)}+\overrightarrow{\mathcal{I}}_{\sigma_{j+1}}^{\sigma_{j}}\right)\right]\left(\frac{1}{K_{\vec{P}}^{\sigma_{j}}-z_{j+1}}\right)^{\frac{1}{2}} \Phi_{\vec{P}}^{\sigma_{j}}\right\|
$$

(where $\overrightarrow{\mathcal{L}}_{\sigma_{j+1}}^{\sigma_{j}(+)}$ stands for the part which contains only photon creation operators) is of order $\mathcal{O}\left(\epsilon^{\eta j}\right)$, for some $\eta>0$ specified in [6]. In particular, this suffices to show that

$$
\left\|\hat{\Phi}_{\vec{P}}^{\sigma_{j+1}}-\Phi_{\vec{P}}^{\sigma_{j}}\right\| \leq \mathcal{O}\left(\epsilon^{\frac{j+1}{2}(1-\delta)}\right)
$$

for any $0<\delta<1$ provided $\alpha$ is sufficiently small. Finally, in Theorem 3.1 of ref. [6], it is proven that there is a non-zero vector in the Hilbert space corresponding to $\lim _{j \rightarrow \infty} \Phi_{\vec{P}}^{\sigma_{j}}$, and that the rate of convergence is at least $\mathcal{O}\left(\sigma^{\frac{1}{2}(1-\delta)}\right)$ for any $0<\delta<1$ provided $\alpha$ is sufficiently small.

Remark. In Theorem 3.1 of ref. [6], for $\lim _{j \rightarrow \infty} \Phi_{\vec{P}}^{\sigma_{j}}$, the range of values of $\alpha$ such that the rate of convergence, $\mathcal{O}\left(\sigma^{\frac{1}{2}(1-\delta)}\right)$, holds is not claimed to be uniform in $\delta$. The stronger result obtained in the next section (see (III.3) and (III.34)) implies that this range (corresponding to the rate $\mathcal{O}\left(\sigma^{\frac{1}{2}(1-\delta)}\right)$ ) is actually $\delta$-independent.

II.3.1. Key ingredients. To prove convergence of the sequence $\left\{\Phi_{\vec{P}}^{\sigma_{j}}\right\}$ of ground state vectors of the Hamiltonians $K_{\vec{P}}^{\sigma_{j}}$, some further conditions on $\alpha, \epsilon$, and $\mu$ (see (II.8), (II.9)) are required, in particular an upper bound on $\mu$ and an upper bound on $\epsilon$ strictly smaller than the ones imposed by inequalities (II.8), (II.9) (for details, see Lemma A.3 in [6]). We note that the more restrictive conditions on $\mu$ and $\epsilon$ imply new bounds on $\rho^{-}$and $\rho^{+}$. Moreover, $\epsilon$ must satisfy a lower bound $\epsilon>C \alpha^{\frac{1}{2}}$, with a multiplicative constant $C>0$ sufficiently large.

Some key inequalities needed in our analysis of the convergence properties of $\left\{\Phi_{\vec{P}}^{\sigma_{j}}\right\}$ are summarized below. They will be marked by the symbol $(\mathcal{B})$. In order to reach some important improvements in our estimates of the convergence rate of $\Phi_{\vec{P}}^{\sigma_{j}}$, as $j \rightarrow \infty$ (discussed in the next section), a refined estimate is needed that is stated in $(\mathcal{B 2})$, and a new inequality, see $(\mathcal{B} 5)$, (analogous to $(\mathcal{B} 3)$ and $(\mathcal{B} 4))$ is required.

- Estimates on the shift of the ground state energy and its gradient. There are constants $C_{1}, C_{2}^{\prime}$ such that the following inequalities hold: $(\mathcal{B} 1)$

$$
\left|E_{\vec{P}}^{\sigma_{j}}-E_{\vec{P}}^{\sigma_{j+1}}\right| \leq C_{1} \alpha \epsilon^{j},
$$

see $[6]$. 
- $(\mathcal{B} 2)$

$$
\left|\vec{\nabla} E_{\vec{P}}^{\sigma_{j+1}}-\vec{\nabla} E_{\vec{P}}^{\sigma_{j}}\right| \leq C_{2}^{\prime}\left(\left\|\hat{\Phi}_{\vec{P}}^{\sigma_{j+1}}-\Phi_{\vec{P}}^{\sigma_{j}}\right\|+\alpha^{\frac{1}{4}} \epsilon^{j+1}\right) .
$$

This is an improvement over a corresponding estimate in [6]: It can be proven after the results stated in Theorem 3.1 in [6], in particular the uniform bound from below on $\left\langle\Phi_{\vec{P}}^{\sigma_{j}}, \Phi_{\vec{P}}^{\sigma_{j}}\right\rangle,\left\langle\Phi_{\vec{P}}^{\sigma_{j}}, \Phi_{\vec{P}}^{\sigma_{j}}\right\rangle>\frac{2}{3}$, and following the steps in the proof of Lemma A.2 in [6].

- Bounds relating expectations of operators to expectations of their absolute values. There are constants $C_{3}, C_{4}, C_{5}>1$ such that the following inequalities hold:

$(\mathcal{B} 3)$ For $z_{j+1} \in \gamma_{j+1}$,

$$
\begin{aligned}
& \left\langle\left(\Gamma_{\vec{P}}^{\sigma_{j}}\right)^{i} \Phi_{\vec{P}}^{\sigma_{j}},\left|\frac{1}{K_{\vec{P}}^{\sigma_{j}}-z_{j+1}}\right|\left(\Gamma_{\vec{P}}^{\sigma_{j}}\right)^{i} \Phi_{\vec{P}}^{\sigma_{j}}\right\rangle \\
& \quad \leq C_{3}\left|\left\langle\left(\Gamma_{\vec{P}}^{\sigma_{j}}\right)^{i} \Phi_{\vec{P}}^{\sigma_{j}}, \frac{1}{K_{\vec{P}}^{\sigma_{j}}-z_{j+1}}\left(\Gamma_{\vec{P}}^{\sigma_{j}}\right)^{i} \Phi_{\vec{P}}^{\sigma_{j}}\right\rangle\right|,
\end{aligned}
$$

where $\left(\Gamma_{\vec{P}}^{\sigma_{j}}\right)^{i}$ is the $i^{t h}$ component of $\vec{\Gamma}_{\vec{P}}^{\sigma_{j}}$.

(B4) For $z_{j+1} \in \gamma_{j+1}$,

$$
\begin{aligned}
& \left\langle\left(\mathcal{L}_{\sigma_{j+1}}^{\sigma_{j}(+)}\right)^{l}\left(\Gamma_{\vec{P}}^{\sigma_{j}}\right)^{i} \Phi_{\vec{P}}^{\sigma_{j}},\left|\frac{1}{K_{\vec{P}}^{\sigma_{j}}-z_{j+1}}\right|\left(\mathcal{L}_{\sigma_{j+1}}^{\sigma_{j}(+)}\right)^{l}\left(\Gamma_{\vec{P}}^{\sigma_{j}}\right)^{i} \Phi_{\vec{P}}^{\sigma_{j}}\right\rangle \\
& \quad \leq C_{4} \mid\left\langle\left(\mathcal{L}_{\sigma_{j+1}}^{\sigma_{j}(+)}\right)^{l}\left(\Gamma_{\vec{P}}^{\sigma_{j}}\right)^{i} \Phi_{\vec{P}}^{\sigma_{j}}, \frac{1}{K_{\vec{P}}^{\sigma_{j}}-z_{j+1}}\left(\mathcal{L}_{\sigma_{j+1}}^{\sigma_{j}(+)}\right)^{l}\left(\Gamma_{\vec{P}}^{\sigma_{j}}\right)^{i} \Phi_{\vec{P}}^{\sigma_{j}}\right\rangle,
\end{aligned}
$$

where $\left(\mathcal{L}_{\sigma_{j+1}}^{\sigma_{j}(+)}\right)^{l}$ is the $l^{\text {th }}$ component of $\overrightarrow{\mathcal{L}}_{\sigma_{j+1}}^{\sigma_{j}(+)}$.

(B5) For $z_{j+1} \in \gamma_{j+1}$,

$$
\begin{aligned}
& \left\langle\left(\Gamma_{\vec{P}}^{\sigma_{j}}\right)^{i} \Phi_{\vec{P}}^{\sigma_{j}},\left|\frac{1}{K_{\vec{P}}^{\sigma_{j}}-z_{j+1}}\right|^{2}\left(\Gamma_{\vec{P}}^{\sigma_{j}}\right)^{i} \Phi_{\vec{P}}^{\sigma_{j}}\right\rangle \\
& \quad \leq C_{5} \mid\left\langle\left(\Gamma_{\vec{P}}^{\sigma_{j}}\right)^{i} \Phi_{\vec{P}}^{\sigma_{j}},\left(\frac{1}{K_{\vec{P}}^{\sigma_{j}}-z_{j+1}}\right)^{2}\left(\Gamma_{\vec{P}}^{\sigma_{j}}\right)^{i} \Phi_{\vec{P}}^{\sigma_{j}}\right\rangle .
\end{aligned}
$$

To prove $(\mathcal{B} 3)$ and $(\mathcal{B} 4)$, it suffices to exploit the fact that the spectral support (with respect to $\left.K_{\vec{P}}^{\sigma_{j}}\right|_{\mathcal{F}_{\sigma_{j}}}$ and to $K_{\vec{P}}^{\sigma_{j}} \mathcal{F}_{\sigma_{j+1}}$, respectively) of the two vectors $\left(\Gamma_{\vec{P}}^{\sigma_{j}}\right)^{i} \Phi_{\vec{P}}^{\sigma_{j}}$ and $\left(\mathcal{L}_{\sigma_{j+1}}^{\sigma_{j}(+)}\right)^{l}\left(\Gamma_{\vec{P}}^{\sigma_{j}}\right)^{i} \Phi_{\vec{P}}^{\sigma_{j}}$ is strictly above the ground state energy of $K_{\vec{P}}^{\sigma_{j}}$, since they are both orthogonal to the ground state, $\Phi_{\vec{P}}^{\sigma_{j}}$, of this operator. In the proof of bound (B5), it is also required that $\rho^{-}>3 \mu \epsilon$, as will be assumed in the following.

Remarks. (1) The constants $C_{1}, \ldots, C_{5}$ are independent of $\alpha, \epsilon, \mu$, and $j \in \mathbb{N}$, provided that $\alpha, \epsilon$, and $\mu$ are sufficiently small. 
(2) For the convenience of the reader, we recapitulate the relations between the parameters entering the construction:

$$
\begin{gathered}
0<\rho^{-}<\mu<\rho^{+}<1-C_{\alpha}<\frac{2}{3} \\
0<\epsilon<\frac{\rho^{-}}{\rho^{+}} \\
\epsilon>C \alpha^{1 / 2} \\
\rho^{-}>3 \mu \epsilon .
\end{gathered}
$$

Moreover, we stress that the final result is a small coupling result, i.e., valid for small values of $\alpha$, and that, for technical reasons, small values of the parameters $\epsilon, \mu$ within the constraints listed above (that imply more restrictive bounds on $\rho^{-}, \rho^{+}$) are required.

The crucial estimate for the bound on $\hat{\Phi}_{\vec{P}}^{\sigma_{j+1}}-\Phi_{\vec{P}}^{\sigma_{j}}$ obtained in [6] (see (II.71)) is

$$
\left|\left\langle\left(\Gamma_{\vec{P}}^{\sigma_{j}}\right)^{i} \Phi_{\vec{P}}^{\sigma_{j}}, \frac{1}{K_{\vec{P}}^{\sigma_{j}}-z_{j+1}}\left(\Gamma_{\vec{P}}^{\sigma_{j}}\right)^{i} \Phi_{\vec{P}}^{\sigma_{j}}\right\rangle\right| \leq \frac{R_{0}}{\alpha \epsilon^{j \delta}},
$$

where $R_{0}$ is independent of $j$, and $\delta, 0<\delta<1$, can be taken arbitrarily small for $\alpha$ and $\epsilon$ sufficiently small (depending on $\delta$ ). This estimate will be improved in the next section. As a consequence, our estimate of the convergence rate of $\left\{\Phi_{\vec{P}}^{\sigma_{j}}\right\}$ will be improved. As a corollary, the second derivative of $E_{\vec{P}}^{\sigma}$ is proven to converge, as $\sigma \rightarrow 0$.

\section{Improved Estimate of the Convergence Rate of $\left\{\Phi_{\vec{P}}^{\sigma}\right\}$, as $\sigma \rightarrow 0$, and Uniform Bound on the Second Derivative of $E_{\vec{P}}^{\sigma}$.}

Our arguments in Sect. III rely on the results previously proven in [6] and described in Sect. II, which hold for $\alpha$ small enough. Therefore, in the following, we assume the constraints (II.79)-(II.82), and we make use of the estimates on the spectral gaps (see $(\mathcal{A 1})$ in Sect. II.1) and of the bounds $(\mathcal{B} 1)-(\mathcal{B} 5)$ (see Sect. II.3.1).

We also make use of the lower bounds

$$
\left\langle\hat{\Phi}_{\vec{P}}^{\sigma_{j+1}}, \hat{\Phi}_{\vec{P}}^{\sigma_{j+1}}\right\rangle,\left\langle\Phi_{\vec{P}}^{\sigma_{j}}, \Phi_{\vec{P}}^{\sigma_{j}}\right\rangle>\frac{2}{3}
$$

uniformly in $j \in \mathbb{N}_{0}$, which appear in the proof of Theorem 3.1 of ref. [6]. Assuming these bounds we can simplify the proof by induction in the theorem below.

Theorem III.1. For $\alpha, \epsilon$ sufficiently small (depending on $\delta$ ), $\epsilon>C \alpha^{\frac{1}{16}}$ with $C>0$, the inequality

$$
\left|\left\langle\left(\Gamma_{\vec{P}}^{\sigma_{j}}\right)^{i} \Phi_{\vec{P}}^{\sigma_{j}},\left(\frac{1}{K_{\vec{P}}^{\sigma_{j}}-z_{j+1}}\right)^{2}\left(\Gamma_{\vec{P}}^{\sigma_{j}}\right)^{i} \Phi_{\vec{P}}^{\sigma_{j}}\right\rangle\right| \leq \frac{\mathcal{R}_{0}}{\alpha^{\frac{1}{2}} \epsilon^{2 j \delta}}
$$


holds true, where $0<\delta<1$ and $\mathcal{R}_{0}, \mathcal{O}\left(\epsilon^{2}\right)>\mathcal{R}_{0}>\mathcal{O}\left(\alpha^{\frac{3}{8}}\right)$, is independent of $j \in \mathbb{N}_{0}:=\mathbb{N} \cup 0$, and

$$
\left\|\hat{\Phi}_{\vec{P}}^{\sigma_{j}}-\Phi_{\vec{P}}^{\sigma_{j-1}}\right\| \leq \alpha^{\frac{1}{4}} \epsilon^{j(1-\delta)} .
$$

$\mathcal{R}_{0}$ can be taken arbitrarily small provided $\alpha$ is small enough.

Proof by induction. - Inductive hypothesis. We assume that, at scale $j-1(\geq 0)$, the following estimate holds:

$$
\left|\left\langle\left(\Gamma_{\vec{P}}^{\sigma_{j-1}}\right)^{i} \Phi_{\vec{P}}^{\sigma_{j-1}},\left(\frac{1}{K_{\vec{P}}^{\sigma_{j-1}}-z_{j}}\right)^{2}\left(\Gamma_{\vec{P}}^{\sigma_{j-1}}\right)^{i} \Phi_{\vec{P}}^{\sigma_{j-1}}\right\rangle\right| \leq \frac{\mathcal{R}_{0}}{\alpha^{\frac{1}{2}} \epsilon^{2(j-1) \delta}} .
$$

This estimate readily implies that, assuming $\mathcal{R}_{0} / \epsilon^{2}$ and $\alpha$ small enough, uniformly in $j$,

$$
\begin{aligned}
& \left\|\hat{\Phi}_{\vec{P}}^{\sigma_{j}}-\Phi_{\vec{P}}^{\sigma_{j-1}}\right\| \\
& \quad=\left\|\sum_{n=1}^{\infty} \frac{1}{2 \pi i} \oint_{\gamma_{j}} d z_{j} \frac{1}{K_{\vec{P}}^{\sigma_{j-1}}-z_{j}}\left[-\left.\Delta K_{\vec{P}}\right|_{\sigma_{j}^{j}} ^{\sigma_{j-1}} \frac{1}{K_{\vec{P}}^{\sigma_{j-1}}-z_{j}}\right] \Phi_{\vec{P}}^{n}\right\| \\
& \quad \leq \alpha^{\frac{1}{4} \epsilon^{j(1-\delta)}} .
\end{aligned}
$$

An improved estimate on $\left\|\hat{\Phi}_{\vec{P}}^{\sigma_{j}}-\Phi_{\vec{P}}^{\sigma_{j-1}}\right\|$ is based on the following bounds where $\epsilon>C \alpha^{\frac{1}{16}}$ :

i)

$$
\left\|\left.\frac{1}{K_{\vec{P}}^{\sigma_{j-1}}-z_{j}} \Delta K_{\vec{P}}\right|_{\sigma_{j}^{j-1}} ^{\sigma_{j-1}} \Phi_{\vec{P}}^{\sigma_{j-1}}\right\| \leq \mathcal{O}\left(\mathcal{R}_{0}^{\frac{1}{2}} \alpha^{\frac{1}{4}} \epsilon^{(j-1)(1-\delta)}+\alpha \epsilon^{2 j-3}\right),
$$

whose proof requires the use of the "pull-through formula" (see, e.g., [14]), a Neumann expansion of the resolvent, the inequality in Eq. (II.78), and Eq. (III.4); the reader can follow the similar steps used in Lemma A3 of ref. [6];

ii)

$$
\left\|\left.\frac{1}{K_{\vec{P}}^{\sigma_{j-1}}-z_{j}} \Delta K_{\vec{P}}\right|_{\sigma_{j}} ^{\sigma_{j-1}}\right\|_{\mathcal{F}_{\sigma_{j}}} \leq \mathcal{O}\left(\alpha^{\frac{1}{2}} / \epsilon\right)
$$

this estimate can be derived from standard bounds and using the "pull-through formula".

- Induction step from scale $j-1$ to scale $j$. By unitarity of $W_{\sigma_{j}}\left(\vec{\nabla} E_{\vec{P}}^{\sigma_{j-1}}\right) W_{\sigma_{j}}^{*}\left(\vec{\nabla} E_{\vec{P}}^{\sigma_{j}}\right)$, we have that

$$
\begin{aligned}
& \left|\left\langle\left(\Gamma_{\vec{P}}^{\sigma_{j}}\right)^{i} \Phi_{\vec{P}}^{\sigma_{j}},\left(\frac{1}{K_{\vec{P}}^{\sigma_{j}}-z_{j+1}}\right)^{2}\left(\Gamma_{\vec{P}}^{\sigma_{j}}\right)^{i} \Phi_{\vec{P}}^{\sigma_{j}}\right\rangle\right| \\
& \quad=\mid\left\langle\left(\hat{\Gamma}_{\vec{P}}^{\sigma_{j}}\right)^{i} \hat{\Phi}_{\vec{P}}^{\sigma_{j}},\left(\frac{1}{\hat{K}_{\vec{P}}^{\sigma_{j}}-z_{j+1}}\right)^{2}\left(\hat{\Gamma}_{\vec{P}}^{\sigma_{j}}\right)^{i} \hat{\Phi}_{\vec{P}}^{\sigma_{j}}\right\rangle .
\end{aligned}
$$


For $\alpha$ small enough and $\epsilon>C \alpha^{\frac{1}{2}}$, where $C>0$ is large enough, we may use $(\mathcal{B} 1)$ to re-expand the resolvent and find that

$$
\begin{aligned}
& \left|\left\langle\left(\hat{\Gamma}_{\vec{P}}^{\sigma_{j}}\right)^{i} \hat{\Phi}_{\vec{P}}^{\sigma_{j}},\left(\frac{1}{\hat{K}_{\vec{P}}^{\sigma_{j}}-z_{j+1}}\right)^{2}\left(\hat{\Gamma}_{\vec{P}}^{\sigma_{j}}\right)^{i} \hat{\Phi}_{\vec{P}}^{\sigma_{j}}\right\rangle\right| \\
& \quad \leq 2 \mid\left\langle\left(\hat{\Gamma}_{\vec{P}}^{\sigma_{j}}\right)^{i} \hat{\Phi}_{\vec{P}}^{\sigma_{j}},\left|\frac{1}{K_{\vec{P}}^{\sigma_{j-1}}-z_{j+1}}\right|^{2}\left(\hat{\Gamma}_{\vec{P}}^{\sigma_{j}}\right)^{i} \hat{\Phi}_{\vec{P}}^{\sigma_{j}}\right\rangle .
\end{aligned}
$$

It follows that

$$
\begin{aligned}
& 2\left|\left\langle\left(\hat{\Gamma}_{\vec{P}}^{\sigma_{j}}\right)^{i} \hat{\Phi}_{\vec{P}}^{\sigma_{j}},\left|\frac{1}{K_{\vec{P}}^{\sigma_{j-1}}-z_{j+1}}\right|^{2}\left(\hat{\Gamma}_{\vec{P}}^{\sigma_{j}}\right)^{i} \hat{\Phi}_{\vec{P}}^{\sigma_{j}}\right\rangle\right| \\
& \leq 4|| \frac{1}{K_{\vec{P}-1}^{\sigma_{j-1}}-z_{j+1}} \mid\left(\left(\hat{\Gamma}_{\vec{P}}^{\sigma_{j}}\right)^{i} \hat{\Phi}_{\vec{P}}^{\sigma_{j}}-\left(\Gamma_{\vec{P}}^{\sigma_{j-1}}\right)^{i} \Phi_{\vec{P}}^{\sigma_{j-1}}\right) \|^{2} \\
& \quad+4\left|\left\langle\left(\Gamma_{\vec{P}}^{\sigma_{j-1}}\right)^{i} \Phi_{\vec{P}}^{\sigma_{j-1}},\left|\frac{1}{K_{\vec{P}}^{\sigma_{j-1}}-z_{j+1}}\right|^{2}\left(\Gamma_{\vec{P}}^{\sigma_{j-1}}\right)^{i} \Phi_{\vec{P}}^{\sigma_{j-1}}\right\rangle\right|
\end{aligned}
$$

Our recursion, combined with (II.78), relates (III.14) to the initial expression in (III.2), with $j$ replaced by $j-1$, while (III.13) is a remainder term. Next we note that

$$
\begin{aligned}
& 4\left\|\frac{1}{K_{\vec{P}}^{\sigma_{j-1}}-z_{j+1}} \mid\left(\left(\hat{\Gamma}_{\vec{P}}^{\sigma_{j}}\right)^{i} \hat{\Phi}_{\vec{P}}^{\sigma_{j}}-\left(\Gamma_{\vec{P}}^{\sigma_{j-1}}\right)^{i} \Phi_{\vec{P}}^{\sigma_{j-1}}\right)\right\|^{2} \\
& \quad \leq 8\left\|\left|\frac{1}{K_{\vec{P}}^{\sigma_{j-1}}-z_{j+1}}\right|\left(\left(\hat{\Gamma}_{\vec{P}}^{\sigma_{j}}\right)^{i} \hat{\Phi}_{\vec{P}}^{\sigma_{j}}-\left(\Gamma_{\vec{P}}^{\sigma_{j-1}}\right)^{i} \hat{\Phi}_{\vec{P}}^{\sigma_{j}}\right)\right\|^{2} \\
& \quad+8\left\|\left|\frac{1}{K_{\vec{P}}^{\sigma_{j-1}}-z_{j+1}}\right|\left(\Gamma_{\vec{P}}^{\sigma_{j-1}}\right)^{i}\left(\hat{\Phi}_{\vec{P}}^{\sigma_{j}}-\Phi_{\vec{P}}^{\sigma_{j-1}}\right)\right\|^{2} \\
& \leq \frac{\mathcal{R}_{1}}{\epsilon^{2 j \delta}}+\frac{\mathcal{R}_{2}}{\epsilon^{2 j \delta}} .
\end{aligned}
$$

Here $\mathcal{R}_{1} \leq \mathcal{O}\left(\epsilon^{-2}\right)$ and $\mathcal{R}_{2} \leq \mathcal{O}\left(\epsilon^{-2}\right)$ are constants independent of $\alpha, \mu$, and $j \in \mathbb{N}$, provided that $\alpha$ is sufficiently small and $\epsilon>C \alpha^{\frac{1}{16}}$. In detail:

- Property (B4) and the two norm-bounds

$$
\left\|\frac{1}{K_{\vec{P}}^{\sigma_{j-1}}-z_{j+1}}\left(\Gamma_{\vec{P}}^{\sigma_{j-1}}\right)^{i}\right\|_{\mathcal{F}_{\sigma_{j}}} \leq \mathcal{O}\left(\epsilon^{-(j+1)}\right), \quad\left\|\hat{\Phi}_{\vec{P}}^{\sigma_{j}}-\Phi_{\vec{P}}^{\sigma_{j-1}}\right\| \leq \alpha^{\frac{1}{4}} \epsilon^{j(1-\delta)}
$$

(see (III.5)) justify the step from (III.17) to (III.18); 
- Concerning the step from (III.16) to (III.18), it is enough to consider Eq. (II.63) and the two bounds

$$
\left\|\left(\mathcal{L}_{\sigma_{j}}^{\sigma_{j-1}}\right)^{i} \hat{\Phi}_{\vec{P}}^{\sigma_{j}}\right\| \leq \mathcal{O}\left(\alpha^{\frac{1}{2}} \epsilon^{j-1}\right), \quad\left\|\hat{\Phi}_{\vec{P}}^{\sigma_{j}}-\Phi_{\vec{P}}^{\sigma_{j-1}}\right\| \leq \alpha^{\frac{1}{4}} \epsilon^{j(1-\delta)} .
$$

(Hint: for the first inequality in (III.20), use the expression in (II.64).)

To bound the term (III.14), we use (B5) and the key orthogonality property (II.69). For $z_{j} \in \gamma_{j}$ and $z_{j+1} \in \gamma_{j+1}$, we find that for $\epsilon / \rho^{-}$sufficiently small,

$$
\begin{aligned}
& 4\left|\left\langle\left(\Gamma_{\vec{P}}^{\sigma_{j-1}}\right)^{i} \Phi_{\vec{P}}^{\sigma_{j-1}},\left|\frac{1}{K_{\vec{P}}^{\sigma_{j-1}}-z_{j+1}}\right|^{2}\left(\Gamma_{\vec{P}}^{\sigma_{j-1}}\right)^{i} \Phi_{\vec{P}}^{\sigma_{j-1}}\right\rangle\right| \\
& \quad \leq 4 C_{5}\left|\left\langle\left(\Gamma_{\vec{P}}^{\sigma_{j-1}}\right)^{i} \Phi_{\vec{P}}^{\sigma_{j-1}},\left(\frac{1}{K_{\vec{P}}^{\sigma_{j-1}}-z_{j+1}}\right)^{2}\left(\Gamma_{\vec{P}}^{\sigma_{j-1}}\right)^{i} \Phi_{\vec{P}}^{\sigma_{j-1}}\right\rangle\right| \\
& \quad \leq 8 C_{5}^{2} \mid\left\langle\left(\Gamma_{\vec{P}}^{\sigma_{j-1}}\right)^{i} \Phi_{\vec{P}}^{\sigma_{j-1}},\left(\frac{1}{K_{\vec{P}}^{\sigma_{j-1}}-z_{j}}\right)^{2}\left(\Gamma_{\vec{P}}^{\sigma_{j-1}}\right)^{i} \Phi_{\vec{P}}^{\sigma_{j-1}}\right\rangle .
\end{aligned}
$$

In passing from (III.22) to (III.23), we again use the constraint on the spectral support (with respect to $K_{\vec{P}}^{\sigma_{j-1}} \mid \mathcal{F}_{\sigma_{j-1}}$ ) of the vector $\left(\Gamma_{\vec{P}}^{\sigma_{j-1}}\right)^{i} \Phi_{\vec{P}}^{\sigma_{j-1}}$.

Assuming that the parameters $\epsilon$ and $\alpha$ are so small that the previous constraints are fulfilled and that

$$
0<\mathcal{R}_{1}+\mathcal{R}_{2} \leq\left(1-8 C_{5}^{2} \epsilon^{2 \delta}\right) \frac{\mathcal{R}_{0}}{\alpha^{\frac{1}{2}}}
$$

we then conclude that

$$
\begin{aligned}
& \left|\left\langle\left(\Gamma_{\vec{P}}^{\sigma_{j}}\right)^{i} \Phi_{\vec{P}}^{\sigma_{j}},\left(\frac{1}{\hat{K}_{\vec{P}}^{\sigma_{j}}-z_{j+1}}\right)^{2}\left(\Gamma_{\vec{P}}^{\sigma_{j}}\right)^{i} \Phi_{\vec{P}}^{\sigma_{j}}\right\rangle\right| \\
& \leq \frac{\mathcal{R}_{1}}{\epsilon^{2 j \delta}}+\frac{\mathcal{R}_{2}}{\epsilon^{2 j \delta}} \\
& \quad+8 C_{5}^{2}\left|\left\langle\left(\Gamma_{\vec{P}}^{\sigma_{j-1}}\right)^{i} \Phi_{\vec{P}}^{\sigma_{j-1}},\left(\frac{1}{K_{\vec{P}}^{\sigma_{j-1}}-z_{j}}\right)^{2}\left(\Gamma_{\vec{P}}^{\sigma_{j-1}}\right)^{i} \Phi_{\vec{P}}^{\sigma_{j-1}}\right\rangle\right| \\
& \leq \frac{\mathcal{R}_{0}}{\alpha^{\frac{1}{2}} \epsilon^{2 j \delta}} .
\end{aligned}
$$

Notice that the bound in (III.24) induces a $\delta$-dependent constraint on the admissible values of $\epsilon$ and, indirectly, on $\alpha$. Moreover, the bound in (III.24) is fulfilled if $\left(\mathcal{O}\left(\epsilon^{2}\right)>\right) \mathcal{R}_{0}>\mathcal{O}\left(\alpha^{\frac{3}{8}}\right) \geq \mathcal{O}\left(\frac{\alpha \frac{1}{2}}{\epsilon^{2}}\right)$ as $\alpha \rightarrow 0$.

- The zeroth step in the induction. Since

$$
\left(\Gamma_{\vec{P}}^{\sigma_{0}}\right)^{i} \equiv\left(\vec{P}^{f}\right)^{i} \quad, \quad \Phi_{\vec{P}}^{\sigma_{0}} \equiv \Omega_{f}
$$


inequality (III.2) is trivially fulfilled for $j=0$; thus (III.2) holds for all $j \in \mathbb{N}_{0}$ and for $\mathcal{R}_{0}$ arbitrarily small, provided $\alpha$ is small enough.

As we explain below, an improved estimate of the rate of convergence of the sequence $\left\{\Phi_{\vec{P}}^{\sigma_{j}}\right\}_{j=0}^{\infty}$ follows from the bound in (III.3), but we stress that only the estimates in Eqs. (III.2), (III.3) will be used for the uniform bound on the second derivative of $E_{\vec{P}}^{\sigma}$ in the next section.

In fact, one can combine the bound in Eq. (III.3) with the estimate

$$
\left\|\Phi_{\vec{P}}^{\sigma_{j}}-\widehat{\Phi}_{\vec{P}}^{\sigma_{j}}\right\| \leq C \alpha^{\frac{1}{2}}\left|\vec{\nabla} E_{\vec{P}}^{\sigma_{j-1}}-\vec{\nabla} E_{\vec{P}}^{\sigma_{j}}\right|\left|\ln \left(\epsilon^{j}\right)\right|,
$$

where $C$ is independent of $\alpha, \epsilon, \mu$, and $j \in \mathbb{N}$, provided that $\alpha, \epsilon$, and $\mu$ are sufficiently small. The estimate in (III.30) is obtained starting from the definition in Eq. (II.67) and using the soft photon bound

$$
\left\|b_{\vec{k}, \lambda} \Psi_{\vec{P}}^{\sigma_{j}}\right\| \leq C \alpha^{1 / 2} \frac{\mathbf{1}_{\sigma_{j}, \Lambda}(\vec{k})}{|\vec{k}|^{3 / 2}}
$$

(where $\mathbf{1}_{\sigma_{j}, \Lambda}(\vec{k})$ is the characteristic function of the set $\left\{\vec{k}: \sigma_{j}<|\vec{k}| \leq \Lambda\right\}$ ) that follows from inequality (II.10) and the identity

$$
b_{\vec{k}, \lambda} \Psi_{\vec{P}}^{\sigma_{j}}=-\alpha^{\frac{1}{2}} \frac{\mathbf{1}_{\sigma_{j}, \Lambda}(\vec{k})}{|\vec{k}|^{\frac{1}{2}}} \frac{1}{H_{\vec{P}-\vec{k}}^{\sigma_{j}}+|\vec{k}|-E_{\vec{P}}^{\sigma_{j}}} \vec{\varepsilon}_{\vec{k}, \lambda} \cdot \vec{\nabla}_{\vec{P}} H_{\vec{P}}^{\sigma_{j}} \Psi_{\vec{P}}^{\sigma_{j}},
$$

which is derived in [4] by using a "pull-through argument".

By a standard procedure (see, e.g., [12]), one obtains similar results for the ground state vectors of the $\sigma$-dependent Hamiltonians $K_{\vec{P}}^{\sigma}$, for arbitrary $\sigma>0$. A precise statement concerning the rate of convergence is as follows: The normalized ground state vectors (that, with an abuse of notation, we call $\Phi_{\vec{P}}^{\sigma}$ )

$$
\Phi_{\vec{P}}^{\sigma}:=\frac{\frac{1}{2 \pi i} \oint_{\gamma_{\sigma}} d z \frac{1}{K_{\vec{P}}^{\sigma}-z} \Omega_{f}}{\left\|\frac{1}{2 \pi i} \oint_{\gamma_{\sigma}} \frac{1}{K_{\vec{P}}^{\sigma}-z} \Omega_{f}\right\|}
$$

where $\gamma_{\sigma}:=\left\{z \in \mathbb{C}|| z-E_{\vec{P}}^{\sigma} \mid=\frac{\rho^{-}}{2} \sigma\right\}$, converge strongly to a vector $\Phi_{\vec{P}}$, as $\sigma \rightarrow 0$, with

$$
\left\|\Phi_{\vec{P}}^{\sigma}-\Phi_{\vec{P}}\right\| \leq \mathcal{O}\left(\alpha^{\frac{1}{4}}\left(\frac{\sigma}{\Lambda}\right)^{1-\delta}\right)
$$

for any $0<\delta(<1)$, provided $\alpha$ is in an interval $\left(0, \alpha_{\delta}\right)$, where $\alpha_{\delta}>0, \alpha_{\delta} \rightarrow 0$ as $\delta \rightarrow 0$. The $\delta$-dependence of the interval $\alpha_{\delta}$ is an indirect consequence of the upper bound on $\epsilon$ that must be imposed through (III.24) to implement the proof by induction. The relations (II.79)-(II.82) and $\epsilon>C \alpha^{\frac{1}{16}}$ induce a $\delta$-dependence on the other parameters and in particular on $\alpha$. Another $\delta$-type dependence of the estimated rate of convergence, $\mathcal{O}\left(\left(\frac{\sigma}{\Lambda}\right)^{1-\delta}\right)$, comes from the logarithmic term in (III.30). However, this does not spoil the uniformity in $\delta$ of the interval of admissible values of $\alpha$ but only affects the multiplicative constant on the R.H.S. of (III.34). Moreover, with further work, the estimate in Eq. (III.30) can be improved to remove the logarithmic term. 
III.1. Convergence of the second derivative of the ground state energy $E_{\vec{P}}^{\sigma}$. Because of rotational symmetry we have that $E_{\vec{P}}^{\sigma} \equiv E_{|\vec{P}|}^{\sigma}$. Moreover, $\left(H_{\vec{P}}^{\sigma} \mid \mathcal{F}_{\sigma}\right)_{\vec{P} \in \mathcal{S}}$ is an analytic family of type A in $\vec{P} \in \mathcal{S}$, with an isolated eigenvalue $E_{|\vec{P}|}^{\sigma}$. Thus, the second derivative $\frac{\partial^{2} E_{|\vec{P}|}^{\sigma}}{(\partial|\vec{P}|)^{2}}$ is well defined and

$$
\frac{\partial^{2} E_{|\vec{P}|}^{\sigma}}{(\partial|\vec{P}|)^{2}}=\left.\partial_{i}^{2} E_{|\vec{P}|}^{\sigma}\right|_{\vec{P}=P^{i} \hat{i}}, \quad i=1,2,3,
$$

where $\partial_{i}:=\frac{\partial}{\partial P^{i}}$.

Without loss of generality, the following results are proven for the standard sequence $\left(\sigma_{j}\right)_{j=0}^{\infty}$ of infrared cutoffs. By simple arguments (see [12]), limiting behavior as $\sigma \rightarrow 0$ is shown to be "sequence-independent".

By analytic perturbation theory we have that

$$
\begin{aligned}
& \partial_{i}^{2} E_{|\vec{P}|}^{\sigma_{j}} \mid \vec{P}=P^{i \hat{i}} 1-2\left\langle\frac{1}{2 \pi i} \oint_{\gamma_{j}} \frac{1}{H_{\vec{P}}^{\sigma_{j}}-z_{j}}\left[P^{i}-\left(\beta^{\sigma_{j}}\right)^{i}\right]\right. \\
&\left.\quad \times \frac{1}{H_{\vec{P}}^{\sigma_{j}}-z_{j}} d z_{j} \Psi_{\vec{P}}^{\sigma_{j}},\left[P^{i}-\left(\beta^{\sigma_{j}}\right)^{i}\right] \Psi_{\vec{P}}^{\sigma_{j}}\right\rangle\left.\right|_{\vec{P}=P^{i \hat{i}}},
\end{aligned}
$$

here $\Psi_{\vec{P}}^{\sigma_{j}}$ is the normalized ground state eigenvector of $H_{\vec{P}}^{\sigma_{j}}$.

Next, we make use of the Bogoliubov transformation implemented by $W_{\sigma_{j}}\left(\vec{\nabla} E_{\vec{P}}^{\sigma_{j}}\right)$ to show that

$$
\begin{aligned}
& \left\langle\frac{1}{2 \pi i} \oint_{\gamma_{j}} \frac{1}{H_{\vec{P}}^{\sigma_{j}}-z_{j}}\left[P^{i}-\left(\beta^{\sigma_{j}}\right)^{i}\right] \frac{1}{H_{\vec{P}}^{\sigma_{j}}-z_{j}} d z_{j} \Psi_{\vec{P}}^{\sigma_{j}},\left[P^{i}-\left(\beta^{\sigma_{j}}\right)^{i}\right] \Psi_{\vec{P}}^{\sigma_{j}}\right\rangle \\
& =\frac{1}{\left\|\Phi_{\vec{P}}^{\sigma_{j}}\right\|^{2}}\left\langle\frac{1}{2 \pi i} \oint_{\gamma_{j}} \frac{1}{K_{\vec{P}}^{\sigma_{j}}-z_{j}}\left[P^{i}-W_{\sigma_{j}}\left(\vec{\nabla} E_{\vec{P}}^{\sigma_{j}}\right)\left(\beta^{\sigma_{j}}\right)^{i} W_{\sigma_{j}}^{*}\left(\vec{\nabla} E_{\vec{P}}^{\sigma_{j}}\right)\right]\right. \\
& \left.\quad \times \frac{1}{K_{\vec{P}}^{\sigma_{j}}-z_{j}} d z_{j} \Phi_{\vec{P}}^{\sigma_{j}},\left[P^{i}-W_{\sigma_{j}}\left(\vec{\nabla} E_{\vec{P}}^{\sigma_{j}}\right)\left(\beta^{\sigma_{j}}\right)^{i} W_{\sigma_{j}}^{*}\left(\vec{\nabla} E_{\vec{P}}^{\sigma_{j}}\right)\right] \Phi_{\vec{P}}^{\sigma_{j}}\right),
\end{aligned}
$$

where $\Phi_{\vec{P}}^{\sigma_{j}}$ is the ground state eigenvector of $K_{\vec{P}}^{\sigma_{j}}$ (iteratively constructed in Sect. II).

Recalling the definitions

$$
\begin{gathered}
\vec{\Pi}_{\vec{P}}^{\sigma_{j}}:=W_{\sigma_{j}}\left(\vec{\nabla} E_{\vec{P}}^{\sigma_{j}}\right) \vec{\beta}^{\sigma_{j}} W_{\sigma_{j}}^{*}\left(\vec{\nabla} E_{\vec{P}}^{\sigma_{j}}\right)-\left\langle W_{\sigma_{j}}\left(\vec{\nabla} E_{\vec{P}}^{\sigma_{j}}\right) \vec{\beta}^{\sigma_{j}} W_{\sigma_{j}}^{*}\left(\vec{\nabla} E_{\vec{P}}^{\sigma_{j}}\right)\right\rangle_{\Omega_{f}}, \\
\vec{\Gamma}_{\vec{P}}^{\sigma_{j}}:=\vec{\Pi}_{\vec{P}}^{\sigma_{j}}-\left\langle\vec{\Pi}_{\vec{P}}^{\sigma_{j}}\right\rangle_{\Phi_{\vec{P}}}^{\sigma_{j},}
\end{gathered}
$$

and because of the identity (Feynman-Hellman, see (II.36))

$$
\begin{aligned}
\left\langle\vec{\beta}^{\sigma_{j}}\right\rangle_{\psi_{\vec{P}}^{\sigma_{j}}} & =\vec{P}-\vec{\nabla} E_{\vec{P}}^{\sigma_{j}} \\
& =\left\langle\vec{\Pi} \vec{P}_{\vec{P}}^{\sigma_{j}}\right\rangle_{\Phi_{\vec{P}}^{\sigma_{j}}}+\left\langle W_{\sigma_{j}}\left(\vec{\nabla} E_{\vec{P}}^{\sigma_{j}}\right) \vec{\beta}^{\sigma_{j}} W_{\sigma_{j}}^{*}\left(\vec{\nabla} E_{\vec{P}}^{\sigma_{j}}\right)\right\rangle_{\Omega_{f}},
\end{aligned}
$$


we find that

$$
P^{i}-W_{\sigma_{j}}\left(\vec{\nabla} E_{\vec{P}}^{\sigma_{j}}\right)\left(\beta^{\sigma_{j}}\right)^{i} W_{\sigma_{j}}^{*}\left(\vec{\nabla} E_{\vec{P}}^{\sigma_{j}}\right)=-\left(\Gamma_{\vec{P}}^{\sigma_{j}}\right)^{i}+\partial_{i} E_{\vec{P}}^{\sigma_{j}}
$$

hence,

$$
\begin{aligned}
& \left.\partial_{i}^{2} E_{|\vec{P}|}^{\sigma_{j}}\right|_{\vec{P}=P^{i} \hat{i}} \\
& =1-2 \frac{1}{\left\|\Phi_{\vec{P}}^{\sigma_{j}}\right\|^{2}}\left\langle\frac{1}{2 \pi i} \oint_{\gamma_{j}} \frac{1}{K_{\vec{P}}^{\sigma_{j}}-z_{j}}\left[\partial_{i} E_{\vec{P}}^{\sigma_{j}}-\left(\Gamma_{\vec{P}}^{\sigma_{j}}\right)^{i}\right] \frac{1}{K_{\vec{P}}^{\sigma_{j}}-z_{j}} d z_{j} \Phi_{\vec{P}}^{\sigma_{j}},\right. \\
& \left.\quad\left[\partial_{i} E_{\vec{P}}^{\sigma_{j}}-\left(\Gamma_{\vec{P}}^{\sigma_{j}}\right)^{i}\right] \Phi_{\vec{P}}^{\sigma_{j}}\right\rangle\left.\right|_{\vec{P}=P^{i} \hat{i}} .
\end{aligned}
$$

Using the eigenvalue equation

$$
K_{\vec{P}}^{\sigma_{j}} \Phi_{\vec{P}}^{\sigma_{j}}=E_{\vec{P}}^{\sigma_{j}} \Phi_{\vec{P}}^{\sigma_{j}},
$$

the terms proportional to $\left(\partial_{i} E_{\vec{P}}^{\sigma_{j}}\right)^{2}$ and to the mixed terms - i.e., proportional to the product of $\partial_{i} E_{\vec{P}}^{\sigma_{j}}$ and $\left(\Gamma_{\vec{P}}^{\sigma_{j}}\right)^{i}-$ are seen to be identically 0 , because the contour integral vanishes for each $i=1,2,3$, e.g.,

$$
\begin{gathered}
\oint_{\gamma_{j}}\left\langle\frac{1}{K_{\vec{P}}^{\sigma_{j}}-z_{j}}\left[\partial_{i} E_{\vec{P}}^{\sigma_{j}}\right] \frac{1}{K_{\vec{P}}^{\sigma_{j}}-z_{j}} \Phi_{\vec{P}}^{\sigma_{j}},\left[\partial_{i} E_{\vec{P}}^{\sigma_{j}}\right] \Phi_{\vec{P}}^{\sigma_{j}}\right\rangle d \bar{z}_{j} \\
\quad=\oint_{\gamma_{j}}\left\langle\Phi_{\vec{P}}^{\sigma_{j}}, \Phi_{\vec{P}}^{\sigma_{j}}\right\rangle\left(\frac{\partial_{i} E_{\vec{P}}^{\sigma_{j}}}{E_{\vec{P}}^{\sigma_{j}}-\bar{z}_{j}}\right)^{2} d \bar{z}_{j}=0 .
\end{gathered}
$$

It follows that

$$
\begin{aligned}
& \left.\partial_{i}^{2} E_{|\vec{P}|}^{\sigma_{j}}\right|_{\vec{P}=P^{i} \hat{i}} \\
& =1+\left.\frac{1}{\pi i} \oint_{\gamma_{j}} d \bar{z}_{j}\left\langle\frac{1}{K_{\vec{P}}^{\sigma}-z_{j}}\left(\Gamma_{\vec{P}}^{\sigma_{j}}\right)^{i} \frac{1}{K_{\vec{P}}^{\sigma}-z_{j}} \frac{\Phi_{\vec{P}}^{\sigma_{j}}}{\left\|\Phi_{\vec{P}}^{\sigma_{j}}\right\|},\left(\Gamma_{\vec{P}}^{\sigma_{j}}\right)^{i} \frac{\Phi_{\vec{P}}^{\sigma_{j}}}{\left\|\Phi_{\vec{P}}^{\sigma_{j}}\right\|}\right\rangle\right|_{\vec{P}=P^{i} \hat{i}} \\
& =1+\left.\frac{1}{\pi i} \oint_{\gamma_{j}} d \bar{z}_{j} \frac{1}{E_{\vec{P}}^{\sigma_{j}}-\bar{z}_{j}}\left\langle\left(\Gamma_{\vec{P}}^{\sigma_{j}}\right)^{i} \frac{1}{K_{\vec{P}}^{\sigma_{j}}-z_{j}}\left(\Gamma_{\vec{P}}^{\sigma_{j}}\right)^{i} \frac{\Phi_{\vec{P}}^{\sigma_{j}}}{\left\|\Phi_{\vec{P}}^{\sigma_{j}}\right\|}, \frac{\Phi_{\vec{P}}^{\sigma_{j}}}{\left\|\Phi_{\vec{P}}^{\sigma_{j}}\right\|}\right\rangle\right|_{\vec{P}=P^{i} \hat{i}} .
\end{aligned}
$$

We are now ready for the key estimate.

Lemma III.2. The estimate below holds true $(j \in \mathbb{N})$ :

$$
\begin{aligned}
& \mid \oint_{\gamma_{j-1}}\left\langle\left(\Gamma_{\vec{P}}^{\sigma_{j-1}}\right)^{i} \frac{\Phi_{\vec{P}}^{\sigma_{j-1}}}{\left\|\Phi_{\vec{P}}^{\sigma_{j-1}}\right\|}, \frac{1}{K_{\vec{P}}^{\sigma_{j-1}}-\bar{z}_{j-1}}\left(\Gamma_{\vec{P}}^{\sigma_{j-1}}\right)^{i} \frac{\Phi_{\vec{P}}^{\sigma_{j-1}}}{\left\|\Phi_{\vec{P}}^{\sigma_{j-1}}\right\|}\right\rangle \frac{1}{E_{\vec{P}}^{\sigma_{j-1}}-\bar{z}_{j-1}} d \bar{z}_{j-1} \\
& \quad-\oint_{\gamma_{j}}\left\langle\left(\Gamma_{\vec{P}}^{\sigma_{j}}\right)^{i} \frac{\Phi_{\vec{P}}^{\sigma_{j}}}{\left\|\Phi_{\vec{P}}^{\sigma_{j}}\right\|}, \frac{1}{K_{\vec{P}}^{\sigma_{j}}-\bar{z}_{j}}\left(\Gamma_{\vec{P}}^{\sigma_{j}}\right)^{i} \frac{\Phi_{\vec{P}}^{\sigma_{j}}}{\left\|\Phi_{\vec{P}}^{\sigma_{j}}\right\|}\right\rangle \frac{1}{E_{\vec{P}}^{\sigma_{j}}-\bar{z}_{j}} d \bar{z}_{j} \mid \leq \epsilon^{j(1-2 \delta)},
\end{aligned}
$$

for any $0<\delta(<1 / 2)$, and for $\alpha$ and $\epsilon\left(>C \alpha^{\frac{1}{16}}\right)$ small enough depending on $\delta$. 
Proof. By unitarity of $W_{\sigma_{j}}\left(\vec{\nabla} E_{\vec{P}}^{\sigma_{j-1}}\right) W_{\sigma_{j}}^{*}\left(\vec{\nabla} E_{\vec{P}}^{\sigma_{j}}\right)$,

$$
\begin{aligned}
& \oint_{\gamma_{j}}\left\langle\left(\Gamma_{\vec{P}}^{\sigma_{j}}\right)^{i} \frac{\Phi_{\vec{P}}^{\sigma_{j}}}{\left\|\Phi_{\vec{P}}^{\sigma_{j}}\right\|}, \frac{1}{K_{\vec{P}}^{\sigma_{j}}-\bar{z}_{j}}\left(\Gamma_{\vec{P}}^{\sigma_{j}}\right)^{i} \frac{\Phi_{\vec{P}}^{\sigma_{j}}}{\left\|\Phi_{\vec{P}}^{\sigma_{j}}\right\|}\right\rangle \frac{1}{E_{\vec{P}}^{\sigma_{j}}-\bar{z}_{j}} d \bar{z}_{j} \\
& \quad=\oint_{\gamma_{j}}\left\langle\left(\hat{\Gamma}_{\vec{P}}^{\sigma_{j}}\right)^{i} \frac{\hat{\Phi}_{\vec{P}}^{\sigma_{j}}}{\left\|\hat{\Phi}_{\vec{P}}^{\sigma_{j}}\right\|}, \frac{1}{\hat{K}_{\vec{P}}^{\sigma_{j}}-\bar{z}_{j}}\left(\hat{\Gamma}_{\vec{P}}^{\sigma_{j}}\right)^{i} \frac{\hat{\Phi}_{\vec{P}}^{\sigma_{j}}}{\left\|\hat{\Phi}_{\vec{P}}^{\sigma_{j}}\right\|}\right\rangle \frac{1}{E_{\vec{P}}^{\sigma_{j}}-\bar{z}_{j}} d \bar{z}_{j} .
\end{aligned}
$$

By assumption, $\alpha$ is so small that the Neumann series expansions of the resolvents below converge in $\mathcal{F}_{\sigma_{j}}^{b}$ :

$$
\begin{aligned}
& \frac{1}{\hat{K}_{\vec{P}}^{\sigma_{j}}-\bar{z}_{j}}=\frac{1}{K_{\vec{P}}^{\sigma_{j-1}}-\bar{z}_{j}}+\Sigma_{1}^{\infty}\left(K_{\vec{P}}^{\sigma_{j-1}}, \bar{z}_{j}\right), \\
& \frac{1}{E_{\vec{P}}^{\sigma_{j}}-\bar{z}_{j}}=\frac{1}{E_{\vec{P}}^{\sigma_{j-1}}-\bar{z}_{j}}+\Delta\left(E_{\vec{P}}^{\sigma_{j-1}}, \bar{z}_{j}\right),
\end{aligned}
$$

where

$$
\Sigma_{1}^{\infty}\left(K_{\vec{P}}^{\sigma_{j-1}}, \bar{z}_{j}\right):=\sum_{l=1}^{\infty} \frac{1}{K_{\vec{P}}^{\sigma_{j-1}}-\bar{z}_{j}}\left[-\left(\left.\Delta K_{\vec{P}}\right|_{\sigma_{j}} ^{\sigma_{j-1}}+\hat{\mathcal{E}}_{\vec{P}}^{\sigma_{j}}-\mathcal{E}_{\vec{P}}^{\sigma_{j-1}}\right) \frac{1}{K_{\vec{P}}^{\sigma_{j-1}}-\bar{z}_{j}}\right]
$$

and $\left.\Delta K_{\vec{P}}\right|_{\sigma_{j}} ^{\sigma_{j-1}}$ is defined in Eq. (II.66),

$$
\Delta\left(E_{\vec{P}}^{\sigma_{j-1}}, \bar{z}_{j}\right):=\frac{1}{E_{\vec{P}}^{\sigma_{j}}-\bar{z}_{j}}\left(E_{\vec{P}}^{\sigma_{j-1}}-E_{\vec{P}}^{\sigma_{j}}\right) \frac{1}{E_{\vec{P}}^{\sigma_{j-1}}-\bar{z}_{j}} .
$$

We proceed by using the obvious identity,

$$
\begin{aligned}
& \oint_{\gamma_{j}}\left\langle\left(\hat{\Gamma}_{\vec{P}}^{\sigma_{j}}\right)^{i} \frac{\hat{\Phi}_{\vec{P}}^{\sigma_{j}}}{\left\|\hat{\Phi}_{\vec{P}}^{\sigma_{j}}\right\|}, \frac{1}{\hat{K}_{\vec{P}}^{\sigma_{j}}-\bar{z}_{j}}\left(\hat{\Gamma}_{\vec{P}}^{\sigma_{j}}\right)^{i} \frac{\hat{\Phi}_{\vec{P}}^{\sigma_{j}}}{\left\|\hat{\Phi}_{\vec{P}}^{\sigma_{j}}\right\|}\right\rangle \frac{1}{E_{\vec{P}}^{\sigma_{j}}-\bar{z}_{j}} d \bar{z}_{j} \\
& =\oint_{\gamma_{j}}\left\langle\left(\hat{\Gamma}_{\vec{P}}^{\sigma_{j}}\right)^{i} \frac{\hat{\Phi}_{\vec{P}}^{\sigma_{j}}}{\left\|\hat{\Phi}_{\vec{P}}^{\sigma_{j}}\right\|}, \frac{1}{K_{\vec{P}}^{\sigma_{j-1}}-\bar{z}_{j}}\left(\hat{\Gamma}_{\vec{P}}^{\sigma_{j}}\right)^{i} \frac{\hat{\Phi}_{\vec{P}}^{\sigma_{j}}}{\left\|\hat{\Phi}_{\vec{P}}^{\sigma_{j}}\right\|}\right\rangle \frac{1}{E_{\vec{P}}^{\sigma_{j-1}}-\bar{z}_{j}} d \bar{z}_{j} \\
& \quad+\oint_{\gamma_{j}}\left\langle\left(\hat{\Gamma}_{\vec{P}}^{\sigma_{j}}\right)^{i} \frac{\hat{\Phi}_{\vec{P}}^{\sigma_{j}}}{\left\|\hat{\Phi}_{\vec{P}}^{\sigma_{j}}\right\|}, \Sigma_{1}^{\infty}\left(K_{\vec{P}}^{\sigma_{j-1}}, \bar{z}_{j}\right)\left(\hat{\Gamma}_{\vec{P}}^{\sigma_{j}}\right)^{i} \frac{\hat{\Phi}_{\vec{P}}^{\sigma_{j}}}{\left\|\hat{\Phi}_{\vec{P}}^{\sigma_{j}}\right\|}\right\rangle \frac{1}{E_{\vec{P}}^{\sigma_{j-1}}-\bar{z}_{j}} d \bar{z}_{j} \\
& \quad+\oint_{\gamma_{j}}\left\langle\left(\hat{\Gamma}_{\vec{P}}^{\sigma_{j}}\right)^{i} \frac{\hat{\Phi}_{\vec{P}}^{\sigma_{j}}}{\left\|\hat{\Phi}_{\vec{P}}^{\sigma_{j}}\right\|}, \frac{1}{\hat{K}_{\vec{P}}^{\sigma_{j}}-\bar{z}_{j}}\left(\hat{\Gamma}_{\vec{P}}^{\sigma_{j}}\right)^{i} \frac{\hat{\Phi}_{\vec{P}}^{\sigma_{j}}}{\left\|\hat{\Phi}_{\vec{P}}^{\sigma_{j}}\right\|}\right\rangle \Delta\left(E_{\vec{P}}^{\sigma_{j-1}}, \bar{z}_{j}\right) d \bar{z}_{j} .
\end{aligned}
$$

Each of the expressions (III.58) and (III.59) can be rewritten by adding and subtracting $\left(\Gamma_{\vec{P}}^{\sigma_{j-1}}\right)^{i} \frac{\Phi_{\vec{P}}^{\sigma_{j-1}}}{\left\|\Phi_{\vec{P}}^{\sigma_{j-1}}\right\|}$. For (III.58) we get 
(III.58)

$$
\begin{aligned}
& =\oint_{\gamma_{j}}\left\langle\left(\Gamma_{\vec{P}}^{\sigma_{j-1}}\right)^{i} \frac{\Phi_{\vec{P}}^{\sigma_{j-1}}}{\left\|\Phi_{\vec{P}}^{\sigma_{j-1}}\right\|}, \frac{1}{K_{\vec{P}}^{\sigma_{j-1}}-\bar{z}_{j}}\left(\Gamma_{\vec{P}}^{\sigma_{j-1}}\right)^{i} \frac{\Phi_{\vec{P}}^{\sigma_{j-1}}}{\left\|\Phi_{\vec{P}}^{\sigma_{j-1}}\right\|}\right\rangle \frac{1}{E_{\vec{P}}^{\sigma_{j-1}}-\bar{z}_{j}} d \bar{z}_{j} \\
& +\oint_{\gamma_{j}}\left\langle\left(\hat{\Gamma}_{\vec{P}}^{\sigma_{j}}\right)^{i} \frac{\hat{\Phi}_{\vec{P}}^{\sigma_{j}}}{\left\|\hat{\Phi}_{\vec{P}}^{\sigma_{j}}\right\|}-\left(\Gamma_{\vec{P}}^{\sigma_{j-1}}\right)^{i} \frac{\Phi_{\vec{P}}^{\sigma_{j-1}}}{\left\|\Phi_{\vec{P}}^{\sigma_{j-1}}\right\|}\right. \\
& \left.\frac{1}{K_{\vec{P}}^{\sigma_{j-1}}-\bar{z}_{j}}\left[\left(\hat{\Gamma}_{\vec{P}}^{\sigma_{j}}\right)^{i} \frac{\hat{\Phi}_{\vec{P}}^{\sigma_{j}}}{\left\|\hat{\Phi}_{\vec{P}}^{\sigma_{j}}\right\|}-\left(\Gamma_{\vec{P}}^{\sigma_{j-1}}\right)^{i} \frac{\Phi_{\vec{P}}^{\sigma_{j-1}}}{\left\|\Phi_{\vec{P}}^{\sigma_{j-1}}\right\|}\right]\right\rangle \frac{1}{E_{\vec{P}}^{\sigma_{j-1}}-\bar{z}_{j}} d \bar{z}_{j} \\
& +\oint_{\gamma_{j}}\left\langle\left(\hat{\Gamma}_{\vec{P}}^{\sigma_{j}}\right)^{i} \frac{\hat{\Phi}_{\vec{P}}^{\sigma_{j}}}{\left\|\hat{\Phi}_{\vec{P}}^{\sigma_{j}}\right\|}-\left(\Gamma_{\vec{P}}^{\sigma_{j-1}}\right)^{i} \frac{\Phi_{\vec{P}}^{\sigma_{j-1}}}{\left\|\Phi_{\vec{P}}^{\sigma_{j-1}}\right\|},\right. \\
& \left.\frac{1}{K_{\vec{P}}^{\sigma_{j-1}}-\bar{z}_{j}}\left(\Gamma_{\vec{P}}^{\sigma_{j-1}}\right)^{i} \frac{\Phi_{\vec{P}}^{\sigma_{j-1}}}{\left\|\Phi_{\vec{P}}^{\sigma_{j-1}}\right\|}\right\rangle \frac{1}{E_{\vec{P}}^{\sigma_{j-1}}-\bar{z}_{j}} d \bar{z}_{j} \\
& +\oint_{\gamma_{j}}\left\langle\left(\Gamma_{\vec{P}}^{\sigma_{j-1}}\right)^{i} \frac{\Phi_{\vec{P}}^{\sigma_{j-1}}}{\left\|\Phi_{\vec{P}}^{\sigma_{j-1}}\right\|}\right. \\
& \left.\frac{1}{K_{\vec{P}}^{\sigma_{j-1}}-\bar{z}_{j}}\left[\left(\hat{\Gamma}_{\vec{P}}^{\sigma_{j}}\right)^{i} \frac{\hat{\Phi}_{\vec{P}}^{\sigma_{j}}}{\left\|\hat{\Phi}_{\vec{P}}^{\sigma_{j}}\right\|}-\left(\Gamma_{\vec{P}}^{\sigma_{j-1}}\right)^{i} \frac{\Phi_{\vec{P}}^{\sigma_{j-1}}}{\| \Phi_{\vec{P}}^{\sigma_{j-1} \|}}\right]\right\rangle \frac{1}{E_{\vec{P}}^{\sigma_{j-1}}-\bar{z}_{j}} d \bar{z}_{j} .
\end{aligned}
$$

The difference in Eq. (III.50) corresponds to the sum of the terms (III.59)-(III.60) and of the terms (III.62)-(III.64). In fact, (III.61) corresponds to the first term in (III.50) after a contour deformation from $\gamma_{j-1}$ to $\gamma_{j}$.

The sum of the remainder terms (III.59), (III.60), and (III.62)-(III.64) can be bounded by $\epsilon^{j(1-2 \delta)}$, for $\epsilon, \mathcal{R}_{0}$ and $\alpha$ small enough but independent of $j$, for any $\vec{P} \in \mathcal{S}$. (We recall that $\mathcal{R}_{0}$ can be taken arbitrarily small, provided $\alpha$ is small enough, and that $\epsilon>C \alpha^{\frac{1}{16}}$.) The details are as follows.

- For (III.62)-(III.64) use the following inequalities

$$
\begin{aligned}
& \left\|\left(\frac{1}{K_{\vec{P}}^{\sigma_{j-1}}-\bar{z}_{j}}\right)\left(\Gamma_{\vec{P}}^{\sigma_{j-1}}\right)^{i} \Phi_{\vec{P}}^{\sigma_{j-1}}\right\| \leq \mathcal{O}\left(\frac{\mathcal{R}_{0}^{\frac{1}{2}}}{\alpha^{\frac{1}{4}} \epsilon^{(j-1) \delta}}\right), \\
& \left\|\left[\left(\hat{\Gamma}_{\vec{P}}^{\sigma_{j}}\right)^{i}-\left(\Gamma_{\vec{P}}^{\sigma_{j-1}}\right)^{i}\right] \hat{\Phi}_{\vec{P}}^{\sigma_{j}}\right\| \leq \mathcal{O}\left(\alpha^{\frac{1}{4}} \epsilon^{j(1-\delta)}+\alpha^{\frac{1}{2}} \epsilon^{j-1}\right), \\
& \left\|\frac{1}{\| K_{\vec{P}}^{\sigma_{j-1}}-\bar{z}_{j}}\right\|_{\mathcal{F}_{\sigma_{j}}} \leq \mathcal{O}\left(\frac{1}{\epsilon^{j}}\right), \\
& \left\|\left(\Gamma_{\vec{P}}^{\sigma_{j-1}}\right)^{i}\left(\hat{\Phi}_{\vec{P}}^{\sigma_{j}}-\Phi_{\vec{P}}^{\sigma_{j-1}}\right)\right\| \leq \mathcal{O}\left(\alpha^{\frac{1}{4}} \epsilon^{j(1-\delta)}\right), \\
& \left\|\hat{\Phi}_{\vec{P}}^{\sigma_{j}}-\Phi_{\vec{P}}^{\sigma_{j-1}}\right\| \leq \alpha^{\frac{1}{4}} \epsilon^{j(1-\delta)} .
\end{aligned}
$$

In order to derive the inequality in Eq. (III.66), one uses Eqs. (II.63), (II.73), and (II.56)-(II.57). 
- For (III.59), after adding and subtracting $\left(\Gamma_{\vec{P}}^{\sigma_{j-1}}\right)^{i} \frac{\Phi_{\vec{P}}^{\sigma_{j-1}}}{\left\|\Phi_{\vec{P}}^{\sigma_{j-1}}\right\|}$, one also has to use (III.8) and that

$$
\begin{aligned}
& \left\|\left[-\left(\left.\Delta K_{\vec{P}}\right|_{\sigma_{j}} ^{\sigma_{j-1}}+\hat{\mathcal{E}}_{\vec{P}}^{\sigma_{j}}-\mathcal{E}_{\vec{P}}^{\sigma_{j-1}}\right)\right] \frac{1}{K_{\vec{P}}^{\sigma_{j-1}}-z_{j}}\left(\Gamma_{\vec{P}}^{\sigma_{j-1}}\right)^{i} \Phi_{\vec{P}}^{\sigma_{j-1}}\right\| \\
& \quad \leq \mathcal{O}\left(\alpha^{\frac{1}{2}} \epsilon^{j-1} \frac{\mathcal{R}_{0}^{\frac{1}{4}}}{\alpha^{\frac{1}{4}} \epsilon^{(j-1) \delta}}\right) .
\end{aligned}
$$

- To bound (III.60), note that

$$
\begin{aligned}
\text { (III.60) }= & -2 \pi i\left\langle\left(\hat{\Gamma}_{\vec{P}}^{\sigma_{j}}\right)^{i} \frac{\hat{\Phi}_{\vec{P}}^{\sigma_{j}}}{\left\|\hat{\Phi}_{\vec{P}}^{\sigma_{j}}\right\|}, \frac{1}{\hat{K}_{\vec{P}}^{\sigma_{j}}-E_{\vec{P}}^{\sigma_{j}}}\left(\hat{\Gamma}_{\vec{P}}^{\sigma_{j}}\right)^{i} \frac{\hat{\Phi}_{\vec{P}}^{\sigma_{j}}}{\left\|\hat{\Phi}_{\vec{P}}^{\sigma_{j}}\right\|}\right\rangle \\
& +2 \pi i\left\langle\left(\hat{\Gamma}_{\vec{P}}^{\sigma_{j}}\right)^{i} \frac{\hat{\Phi}_{\vec{P}}^{\sigma_{j}}}{\left\|\hat{\Phi}_{\vec{P}}^{\sigma_{j}}\right\|}, \frac{1}{\hat{K}_{\vec{P}}^{\sigma_{j}}-E_{\vec{P}}^{\sigma_{j-1}}}\left(\hat{\Gamma}_{\vec{P}}^{\sigma_{j}}\right)^{i} \frac{\hat{\Phi}_{\vec{P}}^{\sigma_{j}}}{\left\|\hat{\Phi}_{\vec{P}}^{\sigma_{j}}\right\|}\right\rangle \\
= & 2 \pi i\left\langle\left(\hat{\Gamma}_{\vec{P}}^{\sigma_{j}}\right)^{i} \frac{\hat{\Phi}_{\vec{P}}^{\sigma_{j}}}{\left\|\hat{\Phi}_{\vec{P}}^{\sigma_{j}}\right\|}, \frac{\left(E_{\vec{P}}^{\sigma_{j-1}}-E_{\vec{P}}^{\sigma_{j}}\right.}{\hat{K}_{\vec{P}}^{\sigma_{j}}-E_{\vec{P}}^{\sigma_{j}}} \frac{1}{\hat{K}_{\vec{P}}^{\sigma_{j}}-E_{\vec{P}}^{\sigma_{j-1}}}\left(\hat{\Gamma}_{\vec{P}}^{\sigma_{j}}\right)^{i} \frac{\hat{\Phi}_{\vec{P}}^{\sigma_{j}}}{\left\|\hat{\Phi}_{\vec{P}}^{\sigma_{j}}\right\|}\right\rangle,
\end{aligned}
$$

where $\left|E_{\vec{P}}^{\sigma_{j-1}}-E_{\vec{P}}^{\sigma_{j}}\right| \leq \mathcal{O}\left(\alpha \epsilon^{j-1}\right)$. Then use the following inequality analogous to (III.65):

$$
\left\|\left(\frac{1}{\hat{K}_{\vec{P}}^{\sigma_{j}}-E_{\vec{P}}^{\sigma_{j}}}\right)\left(\hat{\Gamma}_{\vec{P}}^{\sigma_{j}}\right)^{i} \hat{\Phi}_{\vec{P}}^{\sigma_{j}}\right\| \leq \mathcal{O}\left(\frac{\mathcal{R}_{0}^{\frac{1}{2}}}{\alpha^{\frac{1}{4}} \epsilon^{j \delta}}\right) .
$$

Theorem III.3. For $\alpha$ small enough, $\frac{\partial^{2} E_{|\vec{P}|}^{\sigma}}{(\partial|\vec{P}|)^{2}}$ converges, as $\sigma \rightarrow 0$. The limiting function, $\Sigma_{|\vec{P}|}:=\lim _{\sigma \rightarrow 0} \frac{\partial^{2} E_{|\vec{P}|}^{\sigma}}{(\partial|\vec{P}|)^{2}}$, is Hölder-continuous in $\vec{P} \in \mathcal{S}($ for an exponent $\eta>0)$. The limit

$$
\lim _{\alpha \rightarrow 0} \Sigma_{|\vec{P}|}=1
$$

holds true uniformly in $\vec{P} \in \mathcal{S}$.

Proof. It is enough to prove the result for a fixed choice of a sequence $\left\{\sigma_{j}\right\}_{j=0}^{\infty}$. The estimate in Lemma III.2 implies the existence of $\left.\lim _{j \rightarrow \infty} \partial_{i}^{2} E_{|\vec{P}|}^{\sigma_{j}}\right|_{\vec{P}=P^{i} \hat{i}}$.

We now observe that $\left.\partial_{i}^{2} E_{|\vec{P}|}^{\sigma_{0}}\right|_{\vec{P}=P^{i} \hat{i}}=1$ (see Eq. (III.49)), because

$$
\left(\Gamma_{\vec{P}}^{\sigma_{0}}\right)^{i} \equiv\left(\vec{P}^{f}\right)^{i} \quad, \quad \Phi_{\vec{P}}^{\sigma_{0}} \equiv \Omega_{f}
$$


According to the constraints in Theorem III.1, we can pick $\epsilon=\mathcal{O}\left(\alpha^{\nu}\right)$ for some small exponent $v>0$ so that, for $\alpha$ small enough, Lemma III.2 and (III.76) yield

$$
\begin{aligned}
& \left|\frac{1}{\pi i} \oint_{\gamma_{j}} d \bar{z}_{j} \frac{1}{E_{\vec{P}}^{\sigma_{j}}-\bar{z}_{j}}\left\langle\frac{\Phi_{\vec{P}}^{\sigma_{j}}}{\left\|\Phi_{\vec{P}}^{\sigma_{j}}\right\|},\left(\Gamma_{\vec{P}}^{\sigma_{j}}\right)^{i} \frac{1}{K_{\vec{P}}^{\sigma_{j}}-\bar{z}_{j}}\left(\Gamma_{\vec{P}}^{\sigma_{j}}\right)^{i} \frac{\Phi_{\vec{P}}^{\sigma_{j}}}{\left\|\Phi_{\vec{P}}^{\sigma_{j}}\right\|}\right\rangle\right|_{\vec{P}=P^{i \hat{i}}} \mid \\
& \quad \leq \mathcal{O}\left(\alpha^{\nu(1-2 \delta)}\right),
\end{aligned}
$$

uniformly in $j \in \mathbb{N}$. Hence the limit (III.75) follows.

The Hölder-continuity in $\vec{P}$ of $\Sigma_{|\vec{P}|}$ is a trivial consequence of the analyticity in $\vec{P} \in \mathcal{S}$ of $E_{\vec{P}}^{\sigma}$, for any $\sigma>0$, and of Lemma III.2; see [12] for similar results.

Corollary III.4. For $\alpha$ small enough, the function $E_{\vec{P}}:=\lim _{\sigma \rightarrow 0} E_{\vec{P}}^{\sigma}, \vec{P} \in \mathcal{S}$, is twice differentiable, and

$$
\frac{\partial^{2} E_{|\vec{P}|}}{(\partial|\vec{P}|)^{2}}=\Sigma_{|\vec{P}|} .
$$

Proof. The result follows from the Hölder-continuity of $\Sigma_{|\vec{P}|}$, of $\lim _{\sigma \rightarrow 0} \frac{\partial E_{|\vec{P}|}^{\sigma}}{\partial|\vec{P}|}$, and from the fundamental theorem of calculus applied to the functions $E_{\vec{P}}$ and $\lim _{\sigma \rightarrow 0} \frac{\partial E_{|\vec{P}|}^{\sigma}}{\partial|\vec{P}|}$, because

$$
\frac{\partial E_{|\vec{P}|}^{\sigma}}{\partial|\vec{P}|} \text { and } \frac{\partial^{2} E_{|\vec{P}|}^{\sigma}}{(\partial|\vec{P}|)^{2}}
$$

converge pointwise, for $\vec{P} \in \mathcal{S}$, as $\sigma \rightarrow 0$,

$$
\left|\frac{\partial E_{|\vec{P}|}^{\sigma}}{\partial|\vec{P}|}\right| \text { and }\left|\frac{\partial^{2} E_{|\vec{P}|}^{\sigma}}{(\partial|\vec{P}|)^{2}}\right|
$$

are uniformly bounded in $\sigma$, for all $\vec{P} \in \mathcal{S}$.

Acknowledgements. The authors thank Thomas Chen for very useful discussions. A.P. was supported by NSF grant DMS-0905988.

Open Access This article is distributed under the terms of the Creative Commons Attribution Noncommercial License which permits any noncommercial use, distribution, and reproduction in any medium, provided the original author(s) and source are credited. 


\section{References}

1. Bach, V., Froehlich, J., Pizzo, A.: Infrared-finite algorithms in QED II. The expansion of the groundstate of an atom interacting with the quantized radiation field. Adv. Math. 220(4), 1023-1074 (2009)

2. Bach, V., Chen, T., Fröhlich, J., Sigal, I.M.: The renormalized electron mass in non-relativistic quantum electrodynamics. J. Funct. Anal. 243(2), 426-535 (2007)

3. Chen, T.: Infrared renormalization in nonrelativistic QED and scaling criticality. J. Funct. Anal. 254(10), 2555-2647 (2008)

4. Chen, T., Fröhlich, J.: Coherent Infrared Representations in Nonrelativistic QED. Proc. Symp. Pure Math. 76 (B. Simon 60-th Birthday Volume), Providence, RI: Amer. Math. Soc., 2007, pp. 25-46

5. Chen, T., Fröhlich, J., Pizzo, A.: Infraparticle scattering states in non-relativistic QED: I. the bloch-nordsieck paradigm. http://arxiv.org/abs/07092493v2[math-ph], 2009

6. Chen, T., Fröhlich, J., Pizzo, A.: Infraparticle scattering states in non-relativistic QED: II. mass hell properties. J. Math. Phys. 50, 012103 (2009)

7. Fierz, M., Pauli, W.: Nuovo cimento 15, 167 (1938)

8. Hiroshima, F., Spohn, H.: Mass renormalization in non-relativistic quantum electrodynamics. J. Math. Phys. 46(4) (2005)

9. Hainzl, C., Seiringer, R.: Mass renormalization and energy level shift in non-relativistic QED. Adv. Theor. Math. Phys. 6(5), 847-871 (2003)

10. Lieb, E.T., Loss, M.: Self Energy of Electrons in Non-perturbative QED. Conference Moshe Flato 1999, Vol. I, Dijon, Math. Phys. Stud. 21, Dordrecht: Kluwer Acad. Publ., 2000, pp. 327-344

11. Lieb, E.T., Loss, M.: A bound on binding energies and mass renormalization in model of quantum electrodynamics. J. Stat. Phys. 108, 1057-1069 (2002)

12. Pizzo, A.: One-particle (improper) states in Nelson's massless model. Ann. H. Poincaré 4(3), 439-486 (2003)

13. Pizzo, A.: Scattering of an Infraparticle: The One-particle (improper) Sector in Nelson's massless model. Ann. H. Poincaré 4(3), 439-486 (2003)

14. Schweber, S.S.: An introduction to Relativistic Quantum Field Theory. New York: Harper and Row, 1961

15. Reed, M., Simon, B.: Methods of Modern Mathematical Physics. Vol. I-II-III-IV, New York: Academic Press, 1972, 1975, 1977, 1978

Communicated by H. Spohn 